\title{
Enhanced One-Pot Microwave Efficient Synthesis of Some Pyrrolidine-2, 5-Diones, Butenamides and their Sulfonamide Derivatives Versus Conventional Thermal Heating Technique
}

\author{
Shadia M. Abdallah
}

Chemistry Department, University College of Women for Arts, Science, and Education, Ain Shams University, Cairo, Egypt.

\begin{abstract}
D UE TO the highly important medical applications and wide biological activities of pyrrolidines, butenamides, and their sulfonamide derivatives, such as bacteriocides, fungicides, and insecticides, compounds (4-42) were synthesized from condensation of $\alpha, \beta$-unsaturated anhydrides (1-3), with the corresponding amines, using either enhancing microwave irradiation heating or the conventional thermal heating technique in order to compare between their efficiency.
\end{abstract}

Keywords: Microwave, Synthesis, Condensation, Butenamides, Pyrrolidines and Sulfonamides.

The microwave irradiation comparable to conventional thermal heating proves to generate rapid intense heating with consequent significant reduction in reaction time, enhancement of yield and purity of products, in addition to its friendly environmental effect, known as green chemistry. It proves to be a convenient method of heating, comparable to conventional thermal technique. Moreover, some reactions that do not take place by conventional thermal heating technique or give very low yields, can be accomplished in high yields under microwave irradiation technique ${ }^{(1,2)}$. Amides, imides, and their derivatives are known to have wide medical applications, biological activities, and as starting materials in industry ${ }^{(3-7)}$. The aim of this work is the comparison between the two techniques and to synthesis amides and imides derivatives in high and pure yield.

\section{Results and Discussion}

\section{Solvent-free microwave irradiation technique}

Solvent-free microwave irradiation of 2-phenylmethylenebutanedioic anhydride (1), 2-(4-methoxyphenylmethylene)butanedioic anhydride (2), or 2 (methylphenylmethylene)butanedioic anhydride $(3)^{(8,9)}$ with different $\mathrm{N}$ substituted 4-aminobenzenesulfonamides $(\mathrm{a}-\mathrm{g})^{(10)}$, gave the corresponding $\mathrm{N}-(\mathrm{N}$ substituted benzenesulfonamido)-3-carboxy-4-aryl-3-butenamide derivatives, and/or N-(N'-substituted benzenesulfonamido)-3-benzylidinepyrrolidine-2,5dione derivatives. The microwave irradiation of anhydride (3) with some amines (h-m) gave the corresponding 3-carboxy-3-butenamide derivatives and/or pyrrolidine-2, 5-dione derivatives. 
The results obtained show that under solvent-free microwave irradiation heating of anhydrides (1 and 2) with N-phenyl-4-aminobenzenesulfonamide (a), $\mathrm{N}$-(4-methylphenyl)-4-aminobenzenesulfonamide (b), N-(4-methoxy- phenyl)-4aminobenzenesulfonamide (c), and N-(4-chlorophenyl)-4-amino- benzenesulfonamide $(\mathrm{d})^{7}$ gave pyrrolidine-2,5-diones (4-7, 12-14 and 16), whereas anhydride (3) gave separable mixtures from the corresponding butenamides (21, 23, 25, and 27) and pyrrolidine-2,5-diones (22, 24, 26, and 28). This can be attributed to the steric effect exerted by the methyl group in anhydride (3).

However, microwave irradiation of anhydrides (1-3) with either N-(4nitrophenyl-4-aminobenzenesulfonamide (e) or N-benzyl-4-aminobenzenesulfonamide $(\mathrm{g})$, gave the corresponding butenamides $(8,11,17,20,29$, and 32). This is ascribed to low nucleophilicity of the amido nitrogen atom towards further intramolecular nucleophilic attack on the carbonyl carbon to give pyrrolidine-2,5-dione, due to the presence of the nitro group, and the steric effect exerted by the $\mathrm{sp}^{3}$ methylene group in the benzyl moiety, respectively.

On the other hand, $\mathrm{N}$-(1-naphthyl)-4-aminobenzenesulfonamide (f) gave with anhydrides (1-3), the respective separable mixtures from butenamides $(9,18$, and 30 ) and pyrrolidine-2, 5-diones (10, 19, and 31). The formation of the cyclic pyrrolidine-2, 5-diones, irrespective to the low reactivity of the amido nitrogen atom in butenamides to form cyclic compounds can be attributed to the coplanarity of the naphthyl ring that facilitates the formation of the cyclic compounds (Scheme 1).

The results obtained from microwave irradiation of anhydride (3) with amines; 4-methylaniline (h), 4-methoxyaniline (i), 4-chloroaniline (j), 4-nitroaniline (k), 1-naphthylamine (l), and benzylamine $(\mathrm{m})$, show that amines $(\mathrm{h}-\mathrm{k})$ gave the corresponding pyrrolidine-2,5-diones $(34,36,38$, and 40), whereas amine (1), gave a separable mixture from butenamide (41) and pyrrolidine- 2,5-dione (42), and amine (m) gave butenamide (43) as an only product (Scheme 2).

Comparison between the results obtained from reaction of anhydride (3) with $\mathrm{N}$-substituted 4-aminobenzenesulfonamides (b-g) versus amines (h-m); shows that the presence of the benzenesulfonamido moiety decreases the reactivity of the amido nitrogen atom towards intramolecular nucleophilic attack to form the cyclic compounds.

\section{Conventional thermal heating technique}

Condensation of anhydrides (1-3) with different 4-amino-N-phenyl-benzenesulfonamide derivatives (a-g) (1:2 mole) in ethanol for at least $4 \mathrm{hr}$ gave the corresponding $\mathrm{N}$-(N'-substituted benzenesulfonamido)-3-carboxy-4-aryl-3butenamide derivatives, and/or $\mathrm{N}$-(N'-substituted benzenesulfonamido)-3benzylidinepyrrolidine-2, 5-dione derivatives. Thermal heating of anhydride (3) with amines (h-m) gave the corresponding 3-carboxy-3-butenamide derivatives and/or pyrrolidine-2, 5-dione derivatives (Scheme 3). 

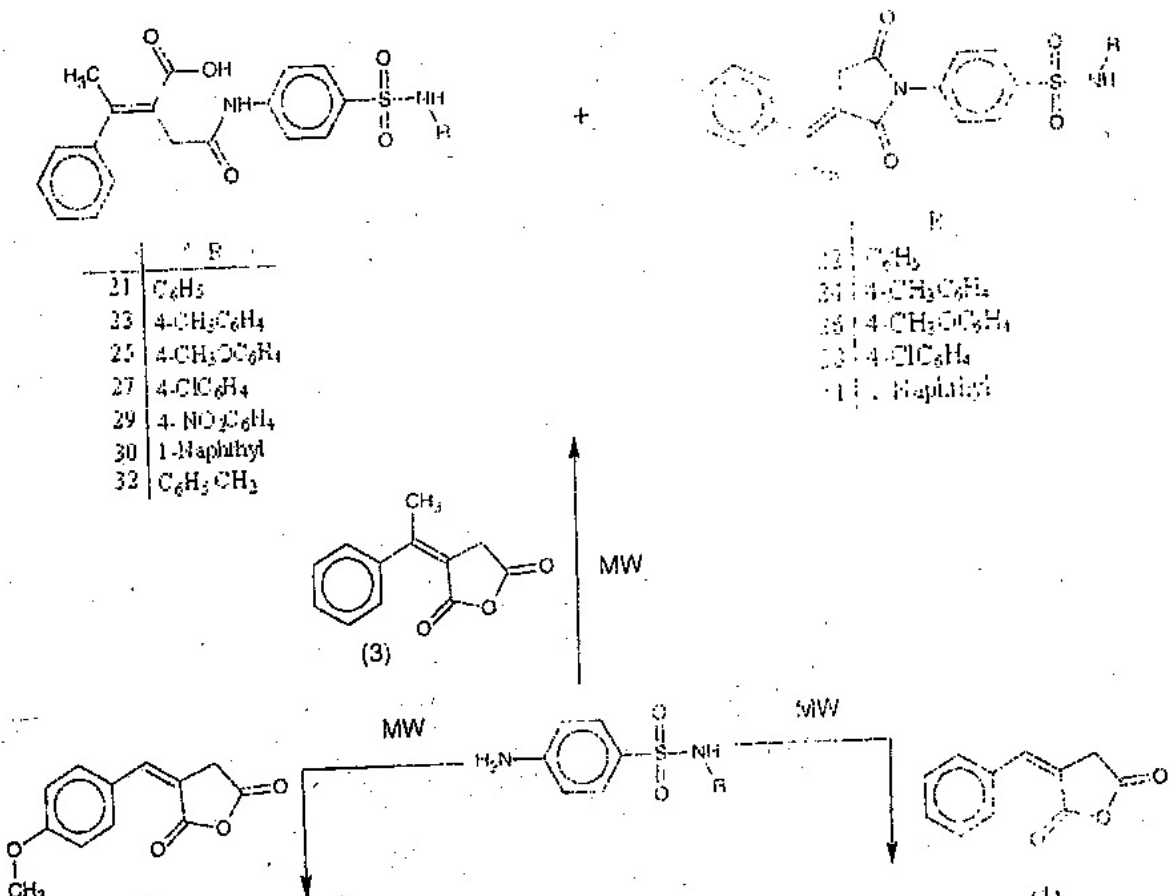

(2)

(i)

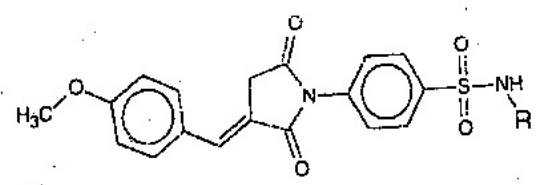<smiles>O=C1C/C(=C\c2ccccc2)C(=O)N1c1ccc(S(=O)(=O)N[Tl])cn1</smiles>

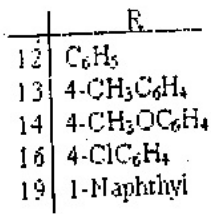

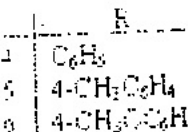

$$
\begin{aligned}
& 7 \mathrm{a}-\mathrm{ClO}_{6} \mathrm{H}_{4}
\end{aligned}
$$

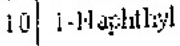
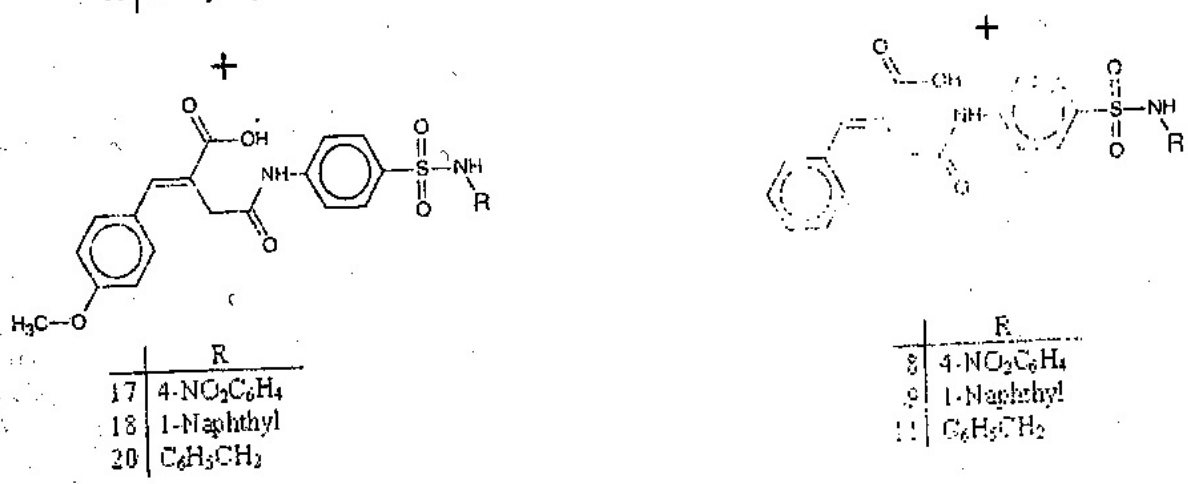

Scheme 1. 


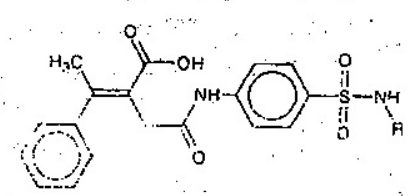

$$
\begin{aligned}
& -\frac{\mathrm{E}}{21} \\
& 2.4 \mathrm{H}_{3} \mathrm{CO}_{\mathrm{C}}
\end{aligned}
$$

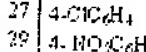

$$
\begin{aligned}
& \text { 3i) } 1+1 \text { andithy } \\
& 32.0 \mathrm{H}_{2} \mathrm{CH}
\end{aligned}
$$

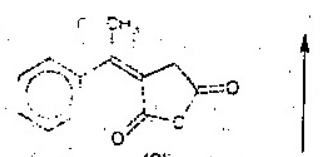

(3)

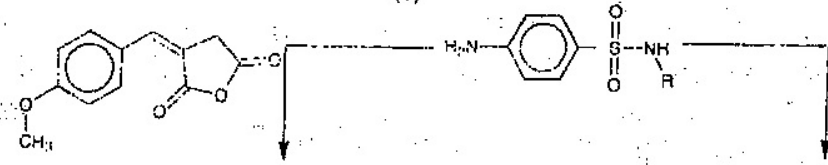<smiles>O=C1C/C(=C/c2ccccc2)C(=O)O1</smiles>

(2)<smiles>[R]NS(=O)(=O)c1ccc([Y](C(=O)/C(=C/C2=IC=C(OC)C=C2)C(C)=O)c2ccc(OC)cc2)cc1</smiles>

$$
21 \frac{\mathrm{F}}{12}
$$$$
13 \text { 4. } \mathrm{C}_{3} \mathrm{H}_{3} \mathrm{C}_{6} \mathrm{H}_{3}
$$

$14 !-4-\mathrm{CH}_{3} \mathrm{CCC}_{6} \mathrm{H}_{4}$

$16 ! 4+10_{6} \mathrm{~S}_{4}$

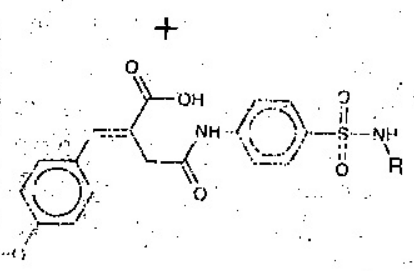

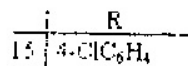

$17 .-\mathrm{HO}_{2} \mathrm{C}_{3} \mathrm{H}_{4}$

18 1. Waphthyl

$20+\mathrm{H}_{3} \mathrm{CH}_{2}$
(1)<smiles>[R]NS(=O)(=O)c1ccc(N2C(=O)C/C(=C\c3ccccc3)C2=O)cc1</smiles>

$$
\begin{array}{l|l} 
& \multicolumn{1}{c}{\mathrm{R}} \\
\hline 4 & \mathrm{C}_{6} \mathrm{H}_{5}^{-1} \\
5 & 4-\mathrm{CH}_{3} \mathrm{C}_{6} \mathrm{H}_{4} \\
6 & 4-\mathrm{CH}_{3} \mathrm{OC}_{6} \mathrm{H}_{4} \\
7 & 4-\mathrm{Cl}_{6} \mathrm{H}_{4}
\end{array}
$$

$+$<smiles>[R]NS(=O)(=O)c1ccc(NC(=O)CC(=Cc2ccccc2)C(=O)O)cc1</smiles>

$$
\begin{aligned}
& \mathrm{R} \\
& 11 \\
& 4-\mathrm{NC}_{2} \mathrm{C}_{6} \mathrm{H}_{4} \\
& 1-\mathrm{Naphthyl}_{3} \\
& \mathrm{C}_{5} \mathrm{H}_{3} \mathrm{CH}_{2}
\end{aligned}
$$

Scheme 3.

Egypt. J. Chem. 53, No. 3 (2010) 
The condensation of anhydrides (1 and 2) with amines (a-c) using conventional thermal heating technique, gave pyrrolidine-2, 5-diones (4-6) and (12-14), respectively. With amine (d), thermal condensation of anhydride (1) gave pyrrolidine-2, 5-dione (7), whereas anhydride (2) gave a separable mixture from butenamide (15) and pyrrolidine-2, 5-dione (16). This can be attributed to the presence of mesomeric effect exerted by the methoxy group in anhydride (2) which decreases the susceptibility of the carbonyl carbon towards the anchimeric attack by the amido nitrogen atom.

On the other hand, the formation of only butenamides $(8,9)$ and $(17,18)$, from the thermal heating of anhydrides ( 1 and 2), respectively, with amines (e- f), can be ascribed to the low nucleophilicity of the amido nitrogen atom in the butenamides $(8,9,17$, and 18$)$, whereas with amine $(\mathrm{g})$, irrespective to the higher nucleophilicity of the amido nitrogen, its reactivity towards cyclization decreases due to the steric effect exerted by the $\mathrm{sp}^{3}$ methylene group.

However, the formation of butenamides $(21,23,25,27$, ,29, 30, 32, 33, 35, $37,39,41$, and 43) as only products, resulted from thermal condensation of anhydride (3) with amines (a-m) can be attributed to the steric effect exerted by the tetrahedral $\mathrm{sp}^{3}$ methyl carbon in anhydride (3), which decreases the susceptibility of the carbonyl carbon to be attacked by the amido nitrogen atom to form the corresponding pyrrolidine-2, 5-dione (Scheme 2)

\section{Experimental}

\section{General solvent-free microwave irradiation technique}

In an open vessel of the anhydride and $\mathrm{N}$-substituted 4-aminobenzenesulfonamide was dry irradiated from 2-5 min in a microwave oven (1000 watt, $100 \%$ power). The reaction progress was monitored by TLC until no more unreacting materials were observed. The reaction mixture was then cooled down to the room temperature and dissolved in chloroform. The chloroform layer was then washed with dilute $\mathrm{HCl}$ to get rid of unreacted amines, and then extracted with $10 \%$ icecold sodium carbonate solution. Acidification of the aqueous layer with ice-cold concentrated hydrochloric acid, precipitated the carboxylic compounds; N- $\left(\mathrm{N}^{\prime}\right.$ substituted benzenesulfonamido)-3-carboxy-4-aryl-3-butenamide derivatives. Thoroughly wash of the organic layer with water followed by its dryness over anhydrous sodium sulfate followed by organic solvent distillation gave $\mathrm{N}-(\mathrm{N}$ substituted benzenesulfonamido)-3-substituted pyrrolidine-2, 5-dione derivatives The products obtained were crystallized from ethanol.

\section{General conventional thermal heating technique}

A mixture of $\alpha, \beta$-unsaturated anhydride (1-3) and N-substituted 4-aminobenzenesulfonamide (1:2) was heated under reflux for at least $4 \mathrm{hr}$ in ethanol. The reaction progress was monitored by thin layer chromatography (TLC). The reaction mixture was then concentrated and the precipitate formed was filtered and dissolved in chloroform then worked up in the same way given in the Egypt. J. Chem. 53, No. 3 (2010) 
solvent-free microwave irradiation technique. Structures of products are confirmed by their spectral analyses, FTIR, ${ }^{1} \mathrm{HNMR}$, and MS.

$N$-(N'-Phenylbenzenesulfonamido)-3-benzylidinepyrrolidine-2, 5-dione (4)

White crystals, m.p. $195^{\circ} \mathrm{C}, 83 \%$ yield in m.w, and $45 \%$ in thermal. FTIR $(\mathrm{KBr}): v\left(\mathrm{~cm}^{-1}\right)=3288(\mathrm{NH}), 1775-1704$ (2CO; imide), 1372 and $1157\left(\mathrm{SO}_{2}\right.$; asy. and sym.). MS: $\mathrm{m} / \mathrm{z}=418\left(\mathrm{M}^{+}, 86.5 \%, \mathrm{C}_{23} \mathrm{H}_{18} \mathrm{~N}_{2} \mathrm{O}_{4} \mathrm{~S}\right), 327\left(12.8, \mathrm{C}_{17} \mathrm{H}_{13} \mathrm{NO}_{2} \mathrm{~S}\right)$, $326\left(46.8, \mathrm{C}_{17} \mathrm{H}_{12} \mathrm{NO}_{4} \mathrm{~S}\right) 263\left(30, \mathrm{C}_{17} \mathrm{H}_{13} \mathrm{NO}_{2}\right), 234\left(31.4, \mathrm{C}_{16} \mathrm{H}_{12} \mathrm{NO}\right), 206(18$, $\left.\mathrm{C}_{15} \mathrm{H}_{12} \mathrm{~N}\right), 143\left(6.5, \mathrm{C}_{10} \mathrm{H}_{7} \mathrm{O}\right), 116\left(34.5, \mathrm{C}_{9} \mathrm{H}_{8}\right), 115\left(100, \mathrm{C}_{9} \mathrm{H}_{7}\right)$, and 97 (5.2, $\mathrm{C}_{4} \mathrm{H}_{3} \mathrm{NO}_{2}$ ).

$N$-[N'-(4-Methylphenyl)benzenesulfonamido]-3-benzylidinepyrrolidine-2,5dione (5)

Yellow crystals, m.p. $238^{\circ} \mathrm{C}, 91 \%$ yield in m.w, and $64 \%$ in thermal. FTIR $(\mathrm{KBr}): v\left(\mathrm{~cm}^{-1}\right)=3271.5(\mathrm{NH}), 1772-1707(2 \mathrm{CO}$; imide $), 1333$ and $1160\left(\mathrm{SO}_{2}\right.$; asy. and sym.). MS: $\mathrm{m} / \mathrm{z}=432\left(\mathrm{M}^{+}, 8.3 \%, \mathrm{C}_{24} \mathrm{H}_{20} \mathrm{~N}_{2} \mathrm{O}_{4} \mathrm{~S}\right), 326$ (1.6, $\left.\mathrm{C}_{17} \mathrm{H}_{12} \mathrm{NO}_{2} \mathrm{~S}\right), 262\left(6.5, \mathrm{C}_{13} \mathrm{H}_{14} \mathrm{~N}_{2} \mathrm{O}_{2} \mathrm{~S}\right), 116\left(40.4, \mathrm{C}_{9} \mathrm{H}_{8}\right), 115\left(64, \mathrm{C}_{9} \mathrm{H}_{7}\right), 107$ $\left(8.8, \mathrm{C}_{7} \mathrm{H}_{9} \mathrm{~N}\right), 106\left(100, \mathrm{C}_{7} \mathrm{H}_{8} \mathrm{~N}\right)$ and $91\left(14.5, \mathrm{C}_{7} \mathrm{H}_{7}\right) .{ }^{1} \mathrm{HNMR}\left(\mathrm{DMSO}-\mathrm{d}_{6}\right): \delta$ $(\mathrm{ppm})=7.62-7.73(3 \mathrm{H}, \mathrm{m} ; \mathrm{Ph}), 7.86-7.93(2 \mathrm{H}, \mathrm{m} ; \mathrm{Ph}), 7.61(1 \mathrm{H}, \mathrm{s} ; \mathrm{C} \underline{\mathrm{H}} \mathrm{Ph}), 3.87$ $\left(2 \mathrm{H}, \mathrm{s} \mathrm{C} \mathrm{CH}_{2} \mathrm{CO}\right), 7.06\left(2 \mathrm{H}, \mathrm{d} ; \mathrm{CH}_{3} \mathrm{Ph}\right), 7.50-7.53(2 \mathrm{H}, \mathrm{d}$; $\mathrm{NHPh}), 10.28(1 \mathrm{H}, \mathrm{s}$, $\left.\mathrm{NHSO}_{2}\right), 7.55-7.58$ [(4H, s; $\left.\mathrm{NHPhSO}_{2}\right)$ and $\left.(1 \mathrm{H}, \mathrm{imp} ; \mathrm{NHCO})\right]$, and $2.15(3 \mathrm{H}, \mathrm{s}$; $\left.\mathrm{CH}_{3} \mathrm{Ph}\right)$.

$N$-[N'-(4-Methoxyphenyl)benzenesulfonamido]-3-benzylidinepyrrolidine-2,5dione (6)

Pale gray crystals, m.p. $185^{\circ} \mathrm{C}, 92 \%$ yield in m.w, and $60 \%$ in thermal. FTIR $(\mathrm{KBr}): v\left(\mathrm{~cm}^{-1}\right)=3264(\mathrm{NH}), 1774-1706$ (2CO; imide), 1333 and $1160\left(\mathrm{SO}_{2}\right.$; asy. and sym.). MS: $\mathrm{m} / \mathrm{z}=448\left(\mathrm{M}^{+}, 48 \%, \mathrm{C}_{24} \mathrm{H}_{20} \mathrm{~N}_{2} \mathrm{O}_{5} \mathrm{~S}\right), 292\left(8.7, \mathrm{C}_{17} \mathrm{H}_{10} \mathrm{NO}_{2} \mathrm{~S}\right), 123$ $\left(9, \mathrm{C}_{7} \mathrm{H}_{9} \mathrm{NO}\right), 122\left(100, \mathrm{C}_{7} \mathrm{H}_{8} \mathrm{NO}\right.$ or $\left.\mathrm{C}_{6} \mathrm{H}_{4} \mathrm{NS}\right), 116\left(5.8, \mathrm{C}_{9} \mathrm{H}_{8}\right)$ and 115 (22.4, $\left.\mathrm{C}_{9} \mathrm{H}_{7}\right)$. ${ }^{1} \mathrm{HNMR}\left(\mathrm{DMSO}-\mathrm{d}_{6}\right): \delta(\mathrm{ppm})=7.48-7.59(3 \mathrm{H}, \mathrm{m} ; \mathrm{Ph}), 7.70-7.82(2 \mathrm{H}, \mathrm{m}$; $\mathrm{Ph}), 7.55(1 \mathrm{H}, \mathrm{s} ; \mathrm{C} \underline{\mathrm{H} h}), 3.87\left(2 \mathrm{H}, \mathrm{s}, \mathrm{C}_{2} \mathrm{CO}\right), 7.59 \quad\left[\left(4 \mathrm{H}, \mathrm{s} ; \mathrm{NHPhSO}_{2}\right)\right.$ and $(1 \mathrm{H}, \mathrm{imp} ; \mathrm{NHCO})], 10.09\left(1 \mathrm{H}, \mathrm{s}, \mathrm{NHSO}_{2}\right), 7.01-7.05(2 \mathrm{H}, \mathrm{d} ; \mathrm{NHPh}), 6.81-6.85$ $\left(2 \mathrm{H}, \mathrm{d} ; \mathrm{PhOCH}_{3}\right)$ and $3.68\left(3 \mathrm{H}, \mathrm{s} ; \mathrm{OCH}_{3}\right)$.

$N$-[N'-(4-Chlorophenyl)benzenesulfonamido]-3-benzylidinepyrrolidine-2,5dione (7)

Pale yellow crystals, m.p. $203^{\circ} \mathrm{C}, 94 \%$ yield in m.w, and $54 \%$ in thermal. FTIR (KBr): $v\left(\mathrm{~cm}^{-1}\right)=3244(\mathrm{NH}), 1771-1711.5(2 \mathrm{CO}$; imide), 1373 and 1157 ( $\mathrm{SO}_{2}$; asy. and sym.). $\mathrm{MS}: \mathrm{m} / \mathrm{z}=452\left(\mathrm{M}^{+}, 86.5 \%, \mathrm{C}_{23} \mathrm{H}_{17} \mathrm{~N}_{2} \mathrm{O}_{4} \mathrm{SCl}\right), 326$ (38.9, $\left.\mathrm{C}_{17} \mathrm{H}_{12} \mathrm{NO}_{4} \mathrm{~S}\right), 262\left(100, \mathrm{C}_{17} \mathrm{H}_{12} \mathrm{NO}_{2}\right), 206\left(12.7, \mathrm{C}_{15} \mathrm{H}_{12} \mathrm{~N}\right), 144\left(6.2, \mathrm{C}_{10} \mathrm{H}_{8} \mathrm{O}\right)$, $127\left(10, \mathrm{C}_{6} \mathrm{H}_{6} \mathrm{NCl}\right), 126\left(32.2, \mathrm{C}_{6} \mathrm{H}_{5} \mathrm{NCl}\right), 125\left(3.2, \mathrm{C}_{6} \mathrm{H}_{4} \mathrm{NCl}\right), 116\left(23.5 \mathrm{C}_{9} \mathrm{H}_{8}\right)$ and $115\left(55, \mathrm{C}_{9} \mathrm{H}_{7}\right) .{ }^{1} \mathrm{HNMR}\left(\right.$ DMSO- $\left._{6}\right): \delta(\mathrm{ppm})=7.70-7.74(3 \mathrm{H}, \mathrm{m} ; \mathrm{Ph})$, 7.90-7.94 (2H, m; Ph), $7.59(1 \mathrm{H}, \mathrm{s} ; \mathrm{CH} \mathrm{Ph}), 3.87\left(2 \mathrm{H}, \mathrm{s}, \mathrm{CH}_{2} \mathrm{CO}\right), 7.59-7.63(2 \mathrm{H}$, d; $\underline{\mathrm{PhNCO}}), 7.49-7.52\left(2 \mathrm{H}, \mathrm{d} ; \mathrm{PhSO}_{2}\right), 10.60\left(1 \mathrm{H}, \mathrm{s}, \underline{\mathrm{NSO}}_{2}\right), 7.14-7.18(2 \mathrm{H}, \mathrm{d}$; $\underline{\mathrm{PhCl}})$ and 7.32-7.36 (2H, d; $\underline{\mathrm{PhNH}})$. 
$N$-[N'-(4-Nitrophenyl)benzenesulfonamido]-3-carboxy-4-phenyl-3-utenamide (8)

Yellow crystals, m.p. $180^{\circ} \mathrm{C}, 94 \%$ yield in m.w, and $47 \%$ in thermal. FTIR $(\mathrm{KBr}): v\left(\mathrm{~cm}^{-1}\right)=3351-3224.5(2 \mathrm{NH}), 3400-2400(\mathrm{OH}$; acid $), 1707.5(\mathrm{CO}$; acid $)$, 1648.5(CO; amide), 1331 and $1151.5\left(\mathrm{SO}_{2}\right.$; asy. and sym.). $\mathrm{MS}: \mathrm{m} / \mathrm{z}=481\left(\mathrm{M}^{+}\right.$, $\left.0 \%, \mathrm{C}_{23} \mathrm{H}_{19} \mathrm{~N}_{3} \mathrm{O}_{7} \mathrm{~S}\right), 463\left(42, \mathrm{C}_{23} \mathrm{H}_{17} \mathrm{~N}_{3} \mathrm{O}_{6} \mathrm{~S}\right), 327$ (9.3, $\left.\mathrm{C}_{17} \mathrm{H}_{13} \mathrm{NO}_{4} \mathrm{~S}\right), 262$ (46.2, $\left.\mathrm{C}_{12} \mathrm{H}_{10} \mathrm{NO}_{3} \mathrm{~S}\right), 156\left(100, \mathrm{C}_{6} \mathrm{H}_{6} \mathrm{NO}_{2} \mathrm{~S}\right), 116\left(17, \mathrm{C}_{9} \mathrm{H}_{8}\right), 115\left(50.3, \mathrm{C}_{9} \mathrm{H}_{7}\right), 92$ (55.6, $\left.\mathrm{C}_{6} \mathrm{H}_{6} \mathrm{~N}\right)$, and $65\left(42, \mathrm{HO}_{2} \mathrm{~S}\right)$.

$N$-[N'-(1-Naphthyl)benzenesulfonamido]-3-carboxy-4-phenyl-3-butenamide (9)

White crystals, m.p. $150^{\circ} \mathrm{C}, 63 \%$ yield in m.w, and $60 \%$ in thermal. FTIR $(\mathrm{KBr})$ : $v\left(\mathrm{~cm}^{-1}\right)=3300-3244(2 \mathrm{NH}), 3400-2400$ (OH; acid), 1700(CO; acid), 1678.1(CO; amide), 1344 and 1158( $\mathrm{SO}_{2}$; asy. and sym. $)$. MS: $\mathrm{m} / \mathrm{z}=468\left(\mathrm{M}^{+}\right.$, $\left.100 \%, \mathrm{C}_{27} \mathrm{H}_{22} \mathrm{~N}_{2} \mathrm{O}_{5} \mathrm{~S}\right), 326\left(28, \mathrm{C}_{17} \mathrm{H}_{12} \mathrm{NO}_{4} \mathrm{~S}\right), 298$ (30, $\left.\mathrm{C}_{16} \mathrm{H}_{14} \mathrm{NO}_{2} \mathrm{~S}\right), 143(37.5$, $\left.\mathrm{C}_{10} \mathrm{H}_{7} \mathrm{O}\right), 142\left(93, \mathrm{C}_{10} \mathrm{H}_{8} \mathrm{~N}\right), 117\left(9.6, \mathrm{C}_{9} \mathrm{H}_{9}\right), 116\left(15.5 \mathrm{C}_{9} \mathrm{H}_{8}\right), 115\left(74, \mathrm{C}_{9} \mathrm{H}_{7}\right)$ and $65\left(8.4, \mathrm{HOS}_{2}\right)$.

$N$-[N'-(1-Naphthyl)benzenesulfonamido]-3-benzylidinepyrrolidine-2,5-dione (10)

Brown crystals, m.p. $89^{\circ} \mathrm{C}, 19 \%$ yield in m.w, and $0 \%$ in thermal. FTIR $(\mathrm{KBr}): v\left(\mathrm{~cm}^{-1}\right)=3247(\mathrm{NH}), 1770.6-1710$ (2CO; imide), 1374 and $1159\left(\mathrm{SO}_{2}\right.$; asy. and sym.). MS: $\mathrm{m} / \mathrm{z}=468\left(\mathrm{M}^{+}, 0 \%, \mathrm{C}_{27} \mathrm{H}_{20} \mathrm{~N}_{2} \mathrm{O}_{4} \mathrm{~S}\right), 314$ (18.5, $\left.\mathrm{C}_{16} \mathrm{H}_{14} \mathrm{~N}_{2} \mathrm{O}_{3} \mathrm{~S}\right), 313\left(79.5, \mathrm{C}_{16} \mathrm{H}_{13} \mathrm{~N}_{2} \mathrm{O}_{3} \mathrm{~S}\right), 298$ (7.7, $\left.\mathrm{C}_{16} \mathrm{H}_{14} \mathrm{~N}_{2} \mathrm{O}_{2} \mathrm{~S}\right), 286(2.5$, $\left.\mathrm{C}_{15} \mathrm{H}_{14} \mathrm{~N}_{2} \mathrm{O}_{2} \mathrm{~S}\right), 285\left(10.4, \mathrm{C}_{15} \mathrm{H}_{13} \mathrm{~N}_{2} \mathrm{O}_{2} \mathrm{~S}\right), 144\left(7, \mathrm{C}_{10} \mathrm{H}_{8} \mathrm{O}\right), 143\left(5, \mathrm{C}_{10} \mathrm{H}_{7} \mathrm{O}\right)$, $116\left(64, \mathrm{C}_{9} \mathrm{H}_{8}\right)$ and $115\left(100, \mathrm{C}_{9} \mathrm{H}_{7}\right)$.

$N$-(N'-Benzylbenzenesulfonamido]-3-carboxy-4-phenyl-3-butenamide (11)

White crystals, m.p. $170^{\circ} \mathrm{C}, 88 \%$ yield in m.w, and $61 \%$ in thermal. FTIR $(\mathrm{KBr}): v\left(\mathrm{~cm}^{-1}\right)=3332.4-3203.3(2 \mathrm{NH}), 3400-2400(\mathrm{OH}$; acid $), 1682.5(\mathrm{CO}$; acid), 1634.5(CO; amide), 1538 and $1156.5\left(\mathrm{SO}_{2}\right.$; asy. and sym.). $\mathrm{MS}: \mathrm{m} / \mathrm{z}=450$ $\left(\mathrm{M}^{+}, 20.7 \%, \mathrm{C}_{24} \mathrm{H}_{22} \mathrm{~N}_{2} \mathrm{O}_{5} \mathrm{~S}\right), 116\left(26.4, \mathrm{C}_{9} \mathrm{H}_{8}\right), 115$ (39, $\left.\mathrm{C}_{9} \mathrm{H}_{7}\right), 108$ (12.7, $\left.\mathrm{C}_{7} \mathrm{H}_{10} \mathrm{~N}\right), 107\left(11, \mathrm{C}_{7} \mathrm{H}_{9} \mathrm{~N}\right), 106\left(100, \mathrm{C}_{7} \mathrm{H}_{8} \mathrm{~N}\right), 91\left(20, \mathrm{C}_{7} \mathrm{H}_{7}\right), 66\left(7.4, \mathrm{H}_{2} \mathrm{O}_{2} \mathrm{~S}\right), 65$ $\left(19, \mathrm{HO}_{2} \mathrm{~S}\right)$ and $64\left(5, \mathrm{O}_{2} \mathrm{~S}\right)$.

${ }^{1} \mathrm{HNMR}\left(\mathrm{DMSO}_{\mathrm{d}}\right): \delta(\mathrm{ppm})=7.77-7.27(3 \mathrm{H}, \mathrm{m} ; \mathrm{Ph}), 7.84-7.78(2 \mathrm{H}, \mathrm{m}$; $\mathrm{Ph}), 7.48$ (1H, s; CHPh), $3.57\left(2 \mathrm{H}, \mathrm{s} ; \mathrm{CH}_{2} \mathrm{CO}\right), 10.53\left(1 \mathrm{H}, \mathrm{s} ; \mathrm{NHSO}_{2}\right), 7.78[4 \mathrm{H}$, s; $\left.\left(4 \mathrm{H}, \mathrm{s} ; \mathrm{NH}^{\mathrm{PhSO}}\right)_{2}\right)$ and $\left.\left.\mathrm{NHCO}\right)\right], 8.06-8.00\left(1 \mathrm{H}, \mathrm{t} ; \mathrm{NHCH}_{2}\right), 3.94-3.97(2 \mathrm{H}, \mathrm{d}$; $\left.\mathrm{CH}_{2} \mathrm{NH}\right), 7.27\left(5 \mathrm{H}, \mathrm{s} ; \underline{\left.\mathrm{PhCH}_{2}\right)}\right.$ and $12.5(1 \mathrm{H}$, broad; $\mathrm{COOH})$.

$N$-(N'-Phenylbenzenesulfonamido)-3-(4-methoxybenzylidine)pyrrolidine-2,5dione (12)

Pale yellow crystals, m.p. $215^{\circ} \mathrm{C}, 91 \%$ yield in m.w, and $67 \%$ in thermal. FTIR (KBr): $v\left(\mathrm{~cm}^{-1}\right)=3242.5(\mathrm{NH}), 1767-1702$ (2CO; imide), 1380 and 1161 ( $\mathrm{SO}_{2}$; asy. and sym.). $\mathrm{MS}: \mathrm{m} / \mathrm{z}=448\left(\mathrm{M}^{+}, 9.1 \%, \mathrm{C}_{24} \mathrm{H}_{20} \mathrm{~N}_{2} \mathrm{O}_{5} \mathrm{~S}\right), 292$ (13, $\left.\mathrm{C}_{18} \mathrm{H}_{14} \mathrm{NO}_{3}\right), 265$ (9, $\left.\mathrm{C}_{17} \mathrm{H}_{15} \mathrm{NO}\right), 146\left(14.4, \mathrm{C}_{10} \mathrm{H}_{10} \mathrm{O}\right), 145\left(14, \mathrm{C}_{10} \mathrm{H}_{9} \mathrm{O}\right), 131$ $\left(12.3, \mathrm{C}_{9} \mathrm{H}_{7} \mathrm{O}\right)$ and $92\left(100, \mathrm{C}_{6} \mathrm{H}_{6} \mathrm{~N}\right) .{ }^{1} \mathrm{HNMR}\left(\mathrm{DMSO}^{-} \mathrm{d}_{6}\right): \delta(\mathrm{ppm})=3.84(3 \mathrm{H}, \mathrm{s}$; $\left.\mathrm{OC}_{3}\right), 7.05-7.09\left(2 \mathrm{H}, \mathrm{d} ; \underline{\mathrm{PhOCH}}{ }_{3}\right), 7.90-7.94(2 \mathrm{H}, \mathrm{d} ; \underline{\mathrm{PhCH}}=), 7.66(1 \mathrm{H}, \mathrm{s}$; 
Cㅂph), 3.2-3.6 (2H, imp.; $\left.\mathrm{CH}_{2} \mathrm{CO}\right), 7.70$ [4H, s; $\left.\mathrm{NPhSO}_{2}\right)$ ], 10.46 (1H, broad; $\mathrm{NHSO}_{2}$ ) and 7.14-7.62 (5H, $\left.\mathrm{m} ; \mathrm{Ph}\right)$.

$N$-[N'-(4-Methylphenyl)benzenesulfonamido]-3-(4-methoxybenzylidine) pyrrolidine 2,5-dione (13)

Yellow crystals, m.p. $220^{\circ} \mathrm{C}, 93 \%$ yield in m.w, and $70 \%$ in thermal. FTIR $(\mathrm{KBr}): v\left(\mathrm{~cm}^{-1}\right)=3276.3(\mathrm{NH}), 1772.5-1706$ (2CO; imide), 1375 and $1161\left(\mathrm{SO}_{2}\right.$; asy and sym.). MS: $\mathrm{m} / \mathrm{z}=462\left(\mathrm{M}^{+}, 0 \%, \mathrm{C}_{25} \mathrm{H}_{22} \mathrm{~N}_{2} \mathrm{O}_{5} \mathrm{~S}\right), 246\left(6.5, \mathrm{C}_{13} \mathrm{H}_{12} \mathrm{NO}_{2} \mathrm{~S}\right)$, $216\left(3.2, \mathrm{C}_{12} \mathrm{H}_{10} \mathrm{NO}_{3}\right), 146\left(12, \mathrm{C}_{10} \mathrm{H}_{10} \mathrm{O}\right), 145\left(6.5, \mathrm{C}_{10} \mathrm{H}_{9} \mathrm{O}\right), 132\left(33.6, \mathrm{C}_{9} \mathrm{H}_{8} \mathrm{O}\right)$, $115\left(18.4, \mathrm{C}_{9} \mathrm{H}_{7}\right), 105\left(100, \mathrm{C}_{7} \mathrm{H}_{7} \mathrm{~N}\right)$ and $104\left(97, \mathrm{C}_{7} \mathrm{H}_{6} \mathrm{~N}\right) .{ }^{1} \mathrm{HNMR}\left(\right.$ DMSO- $\left.\mathrm{d}_{6}\right): \delta$ $(\mathrm{ppm})=3.83\left(3 \mathrm{H}, \mathrm{s} ; \mathrm{OCH}_{3}\right), 7.04-7.09\left(2 \mathrm{H}, \mathrm{d} ; \mathrm{PhOCH}_{3}\right), 7.86-7.90(2 \mathrm{H}, \mathrm{d} ;$ $\underline{\mathrm{PhCH}}=), 7.59(1 \mathrm{H}, \mathrm{s} ; \mathrm{C} \underline{\mathrm{HPh}}), 3.2-3.6\left(2 \mathrm{H}, \mathrm{imp} . ; \mathrm{CH}_{2} \mathrm{CO}\right), 7.55(4 \mathrm{H}, \mathrm{s}$; $\left(\mathrm{NPhSO}_{2}\right), 10.27\left(1 \mathrm{H}, \mathrm{s} ; \mathrm{NHSO}_{2}\right), 7.04-7.09\left[4 \mathrm{H}, \mathrm{s} ; \mathrm{CH}_{3} \underline{\mathrm{PhNH}}\right.$ and $2.20(3 \mathrm{H}, \mathrm{s}$; $\mathrm{PhCH} \underline{H}_{3}$.

$N$-[N'-(4-Methoxyphenyl)benzenesulfonamido]-3-(4-methoxybenzylidine) pyrrolidine2,5-dione (14)

Pale yellow crystals, m.p. $175^{\circ} \mathrm{C}, 95 \%$ yield in m.w, and $71 \%$ in thermal. FTIR (KBr): $v\left(\mathrm{~cm}^{-1}\right)=3281(\mathrm{NH}), 1769-1706$ (2CO; imide), 1377 and 1164 ( $\mathrm{SO}_{2}$; asy. and sym.). MS: $\mathrm{m} / \mathrm{z}=476\left(\mathrm{M}^{+}, 46 \%, \mathrm{C}_{25} \mathrm{H}_{22} \mathrm{~N}_{2} \mathrm{O}_{6} \mathrm{~S}\right), 292$ $\left.\mathrm{C}_{18} \mathrm{H}_{14} \mathrm{NO}_{3}\right), 146\left(5.4, \mathrm{C}_{10} \mathrm{H}_{10} \mathrm{O}\right), 145\left(9.8, \mathrm{C}_{10} \mathrm{H}_{9} \mathrm{O}\right), 131\left(4, \mathrm{C}_{9} \mathrm{H}_{7} \mathrm{O}\right), 123(9$, $\left.\mathrm{C}_{7} \mathrm{H}_{9} \mathrm{NO}\right), 122\left(100, \mathrm{C}_{7} \mathrm{H}_{8} \mathrm{NO}\right), 106\left(2.6, \mathrm{C}_{6} \mathrm{H}_{4} \mathrm{NO}\right), 103\left(6.3, \mathrm{C}_{8} \mathrm{H}_{7}\right)$, and $65(2$, $\mathrm{HOS}_{2}$ ).

$N$-[N'-(4-Chlorophenyl)benzenesulfonamido]-3-carboxy-4-(4-methoxyphenyl)- 3butenamide (15)

White crystals, m.p. $179^{\circ} \mathrm{C}, 0 \%$ yield in m.w, and $47 \%$ in thermal. FTIR $(\mathrm{KBr}): \mathrm{v}\left(\mathrm{cm}^{-1}\right)=3261-3193(2 \mathrm{NH}), 3400-2400(\mathrm{OH} ; \mathrm{acid}), 1690(\mathrm{CO}$, acid $)$, 1667.2 (CO; amide), 1336 and 1157.5( $\mathrm{SO}_{2}$; asy. and sym.). $\mathrm{MS}: \mathrm{m} / \mathrm{z}=500\left(\mathrm{M}^{+}\right.$, $\left.0 \%, \mathrm{C}_{24} \mathrm{H}_{21} \mathrm{~N}_{2} \mathrm{O}_{6} \mathrm{SCl}\right), 327\left(3.2, \mathrm{C}_{16} \mathrm{H}_{11} \mathrm{~N}_{2} \mathrm{O}_{4} \mathrm{~S}\right), 282\left(44, \mathrm{C}_{12} \mathrm{H}_{11} \mathrm{~N}_{2} \mathrm{O}_{2} \mathrm{SCl}\right), 218$ $\left(23.5, \mathrm{C}_{12} \mathrm{H}_{10} \mathrm{O}_{4}\right), 217 \quad\left(4.2, \mathrm{C}_{12} \mathrm{H}_{11} \mathrm{NO}_{3}\right), 191 \quad\left(3.3, \mathrm{C}_{11} \mathrm{H}_{11} \mathrm{O}_{3}\right), 156 \quad(100$, $\left.\mathrm{C}_{6} \mathrm{H}_{6} \mathrm{NO}_{2} \mathrm{~S}\right), 146\left(54, \mathrm{C}_{10} \mathrm{H}_{10} \mathrm{O}\right)$ and $126\left(11.8, \mathrm{C}_{6} \mathrm{H}_{5} \mathrm{NCl}\right) .{ }^{1} \mathrm{HNMR}$ (DMSO-d $\left.\mathrm{d}_{6}\right): \delta$ $(\mathrm{ppm})=3.77\left(3 \mathrm{H}, \mathrm{s} ; \mathrm{OCH}_{3}\right), 7.08-7.11\left(2 \mathrm{H}, \mathrm{d} ; \mathrm{PhOCH}_{3}\right), 7.47-7.43(2 \mathrm{H}, \mathrm{d} ;$ $\underline{\mathrm{PhCH}}=), 3.55\left(2 \mathrm{H}, \mathrm{s} ; \mathrm{CH}_{2} \mathrm{CO}\right), 10.35(1 \mathrm{H}, \mathrm{s} ; \mathrm{NHCO}), 7.77$ [(4H, s; $\left.\mathrm{NPhSO}_{2}\right)$, and (1H,imp.; $\mathrm{CHPh})], 10.55\left(1 \mathrm{H}, \mathrm{s} ; \mathrm{NHSO}_{2}\right), 6.98-7.02(2 \mathrm{H}, \mathrm{d} ; \underline{\mathrm{PhNH}})$ and 7.28-7.32 (2H, d; $\mathrm{PhCl})$.

$N$-[N'-(4-Chlorophenyl)benzenesulfonamido]-3-(4methoxybenzylidine) pyrrolidine2,5-dione (16)

Yellow crystals, m.p. $230^{\circ} \mathrm{C}, 86 \%$ yield in m.w, and $30 \%$ in thermal. FTIR $(\mathrm{KBr}): v\left(\mathrm{~cm}^{-1}\right)=3254(\mathrm{NH}), 1768-1708$ (2CO; imide), 1377 and $1161\left(\mathrm{SO}_{2}\right.$; asy. and sym.). MS: $\mathrm{m} / \mathrm{z}=482\left(\mathrm{M}^{+}, 65.4 \%, \mathrm{C}_{24} \mathrm{H}_{19} \mathrm{~N}_{2} \mathrm{O}_{5} \mathrm{SCl}\right), 356$ (39.1, $\left.\mathrm{C}_{18} \mathrm{H}_{14} \mathrm{NO}_{5} \mathrm{~S}\right), 293\left(27.6, \mathrm{C}_{18} \mathrm{H}_{15} \mathrm{NO}_{3}\right), 292\left(100, \mathrm{C}_{18} \mathrm{H}_{14} \mathrm{NO}_{3}\right), 264$ (33.7, $\left.\mathrm{C}_{12} \mathrm{H}_{7} \mathrm{NO}_{2}\right), 146\left(25.4, \mathrm{C}_{10} \mathrm{H}_{10} \mathrm{O}\right), 145\left(21.3, \mathrm{C}_{10} \mathrm{H}_{9} \mathrm{O}\right), 131\left(21.7, \mathrm{C}_{9} \mathrm{H}_{7} \mathrm{O}\right), 126$ $\left(31, \mathrm{C}_{6} \mathrm{H}_{5} \mathrm{NCl}\right)$ and $115\left(6.5, \mathrm{C}_{9} \mathrm{H}_{7}\right) .{ }^{1} \mathrm{HNMR}\left(\mathrm{DMSO}-\mathrm{d}_{6}\right): \delta(\mathrm{ppm})=3.83(3 \mathrm{H}, \mathrm{s}$; $\left.\mathrm{OCH}_{3}\right), 7.13-7.17\left(2 \mathrm{H}, \mathrm{d} ; \mathrm{PhOCH}_{3}\right), 7.57-7.62(2 \mathrm{H}, \mathrm{d} ; \underline{\mathrm{PhCH}}=), 7.57-7.69(1 \mathrm{H}$, 
imp.; CHPh), 3.2-3.6 (2H, imp.; $\left.\mathrm{CH}_{2} \mathrm{CO}\right), 7.89(2 \mathrm{H}, \mathrm{d} ; \mathrm{PhN}), 7.93(2 \mathrm{H}, \mathrm{d}$; $\left.\underline{\mathrm{PhSO}_{2}}\right), 10.60\left(1 \mathrm{H}, \mathrm{s} ; \mathrm{NHSO}_{2}\right), 7.05-7.09(2 \mathrm{H}, \mathrm{d} ; \underline{\mathrm{PhCl}})$ and 7.32-7.36 $(2 \mathrm{H}, \mathrm{d}$; $\underline{\mathrm{PhNH}}$.

$N$-[N'-(4-Nitrophenyl)benzenesulfonamido]-3-carboxy-4-(4-methoxyphenyl) 3-butenamide (17)

Yellowish brown crystals, m.p. $150{ }^{\circ} \mathrm{C}, 91 \%$ yield in m.w, and $43 \%$ in thermal. FTIR $(\mathrm{KBr}): \mathrm{v}\left(\mathrm{cm}^{-1}\right)=3295-3255(2 \mathrm{NH}), 3400-2400(\mathrm{OH}$, acid), 1690(CO; acid), 1672.3 (CO; amide), 1347 and 1160( $\mathrm{SO}_{2}$; asy. and sym.). MS: $\mathrm{m} / \mathrm{z}=511\left(\mathrm{M}^{+}, 0 \%, \mathrm{C}_{24} \mathrm{H}_{21} \mathrm{~N}_{3} \mathrm{O}_{8} \mathrm{~S}\right), 493 \quad\left(18.6, \quad \mathrm{C}_{24} \mathrm{H}_{19} \mathrm{~N}_{3} \mathrm{O}_{7} \mathrm{~S}\right), 293$ (43.8, $\left.\mathrm{C}_{12} \mathrm{H}_{11} \mathrm{~N}_{3} \mathrm{O}_{4} \mathrm{~S}\right), 218\left(23.4, \mathrm{C}_{12} \mathrm{H}_{10} \mathrm{O}_{4}\right), 156\left(100, \mathrm{C}_{6} \mathrm{H}_{6} \mathrm{NO}_{2} \mathrm{~S}\right), 146\left(28, \mathrm{C}_{19} \mathrm{H}_{10} \mathrm{O}\right)$, $139\left(6.6, \mathrm{C}_{6} \mathrm{H}_{7} \mathrm{~N}_{2} \mathrm{O}_{2}\right), 131\left(8, \mathrm{C}_{9} \mathrm{H}_{7} \mathrm{O}\right), 115\left(6.3, \mathrm{C}_{9} \mathrm{H}_{7}\right)$ and $92\left(94.8, \mathrm{C}_{6} \mathrm{H}_{6} \mathrm{~N}\right)$.

$N$-[N'-(1-Naphthyl) benzenesulfonamido] -3-carboxy-4- (4-methoxyphenyl)-3butenamide (18)

Pale brown crystals, m.p. $170^{\circ} \mathrm{C}, 64 \%$ yield in m.w, and $68 \%$ in thermal. FTIR (KBr): $v\left(\mathrm{~cm}^{-1}\right)=3244-3203(2 \mathrm{NH}), 3400-2400(\mathrm{OH} ;$ acid $), 1717(\mathrm{CO}$; acid), 1667(CO; amide), 1257 and 1176.8( $\mathrm{SO}_{2}$; asy. and sym.). $\mathrm{MS}: \mathrm{m} / \mathrm{z}=516$ $\left(\mathrm{M}^{+}, 0 \%, \mathrm{C}_{28} \mathrm{H}_{24} \mathrm{~N}_{2} \mathrm{O}_{6} \mathrm{~S}\right), 499\left(22.3, \mathrm{C}_{28} \mathrm{H}_{23} \mathrm{~N}_{2} \mathrm{O}_{5} \mathrm{~S}\right), 498$ (17, $\left.\mathrm{C}_{28} \mathrm{H}_{22} \mathrm{~N}_{2} \mathrm{O}_{5} \mathrm{~S}\right), 218$ $\left(65, \mathrm{C}_{12} \mathrm{H}_{10} \mathrm{O}_{4}\right), 191\left(58.8, \mathrm{C}_{11} \mathrm{H}_{11} \mathrm{O}_{3}\right), 147\left(40, \mathrm{C}_{10} \mathrm{H}_{11} \mathrm{O}\right), 146\left(100, \mathrm{C}_{10} \mathrm{H}_{10} \mathrm{O}\right)$, $143\left(38.1 \mathrm{C}_{10} \mathrm{H}_{9} \mathrm{~N}\right), 131\left(47.5, \mathrm{C}_{9} \mathrm{H}_{7} \mathrm{O}\right), 115\left(15.3, \mathrm{C}_{9} \mathrm{H}_{7}\right)$ and $92\left(11.3, \mathrm{C}_{6} \mathrm{H}_{6} \mathrm{~N}\right)$.

$N-\left[N^{\prime}-(1-N a p h t h y l) b e n z e n e s u l f o n a m i d o\right]-3-(4-m e t h o x y b e n z y l i d i n e)$ pyrrolidine 2,5-dione (19)

Reddish brown crystals, m.p. $226^{\circ} \mathrm{C}, 26 \%$ yield in m.w, and $0 \%$ in thermal. FTIR (KBr): $v\left(\mathrm{~cm}^{-1}\right)=3281(\mathrm{NH}), 1704-1706$ (2CO; imide), 1599 and 1126( $\mathrm{SO}_{2}$; asy. and sym.). $\mathrm{MS}: \mathrm{m} / \mathrm{z}=498\left(\mathrm{M}^{+}, 87.6 \%, \mathrm{C}_{28} \mathrm{H}_{22} \mathrm{~N}_{2} \mathrm{O}_{5} \mathrm{~S}\right), 146\left(12.6, \mathrm{C}_{10} \mathrm{H}_{10} \mathrm{O}\right)$, $145\left(9.1, \mathrm{C}_{10} \mathrm{H}_{9} \mathrm{O}\right), 143\left(24.3, \mathrm{C}_{10} \mathrm{H}_{7} \mathrm{O}\right.$ or $\left.\mathrm{C}_{10} \mathrm{H}_{9} \mathrm{~N}\right), 142\left(100, \mathrm{C}_{10} \mathrm{H}_{8} \mathrm{~N}\right), 122(6.2$, $\left.\mathrm{C}_{6} \mathrm{H}_{4} \mathrm{NS}\right), 115\left(53.3, \mathrm{C}_{9} \mathrm{H}_{7}\right)$ and $103\left(9, \mathrm{C}_{8} \mathrm{H}_{7}\right)$.

$N$-[N'-Benzylbenzenesulfonamido]-3-carboxy-4-(4-methoxyphenyl-3-butenamide (20)

Gray crystals, m.p. $190^{\circ} \mathrm{C}, 88 \%$ yield in m.w, and $27 \%$ in thermal. FTIR $(\mathrm{KBr}): v\left(\mathrm{~cm}^{-1}\right)=3371-3260(2 \mathrm{NH}), 3400-2400(\mathrm{OH}$; acid), 1711(CO; acid), 1680 (CO; amide), 1323 and 1154( $\mathrm{SO}_{2}$; asy. and sym.). $\mathrm{MS}: \mathrm{m} / \mathrm{z}=480\left(\mathrm{M}^{+}, 9.1 \%\right.$, $\left.\mathrm{C}_{25} \mathrm{H}_{24} \mathrm{~N}_{2} \mathrm{O}_{6} \mathrm{~S}\right), 293\left(15, \mathrm{C}_{18} \mathrm{H}_{15} \mathrm{NO}_{3}\right), 262\left(12, \mathrm{C}_{13} \mathrm{H}_{14} \mathrm{~N}_{2} \mathrm{O}_{2} \mathrm{~S}\right), 191\left(10, \mathrm{C}_{11} \mathrm{H}_{11} \mathrm{O}_{3}\right)$, $156\left(15 \mathrm{C}_{6} \mathrm{H}_{6} \mathrm{NO}_{2} \mathrm{~S}\right), 146\left(56.5, \mathrm{C}_{10} \mathrm{H}_{10} \mathrm{O}\right), 145\left(39.4, \mathrm{C}_{10} \mathrm{H}_{9} \mathrm{O}\right), 106$ (71.7, $\left.\mathrm{C}_{7} \mathrm{H}_{8} \mathrm{~N}\right), 91\left(84, \mathrm{C}_{7} \mathrm{H}_{7}\right)$ and $77\left(100, \mathrm{C}_{6} \mathrm{H}_{5}\right) .{ }^{1} \mathrm{HNMR}\left(\mathrm{DMSO}-\mathrm{d}_{6}\right): \delta(\mathrm{ppm})=3.79$ $\left(3 \mathrm{H}, \mathrm{s} ; \mathrm{OCH}_{3}\right), 7.00-7.04\left(2 \mathrm{H}, \mathrm{d} ; \underline{\mathrm{PhOCH}}_{3}\right), 7.47-7.50(2 \mathrm{H}, \mathrm{d} ; \mathrm{PhCH}=), 3.59$ $\left(2 \mathrm{H}, \mathrm{s} ; \mathrm{CH}_{2} \mathrm{CO}\right), 10.58(1 \mathrm{H}, \mathrm{s} ; \mathrm{N} \underline{\mathrm{HCO}}), 7.79$ [4H, s; $\left.\left.\mathrm{NH}^{\mathrm{PhSO}}\right)_{2}\right)$ and $(1 \mathrm{H}, \mathrm{imp}$, $\mathrm{PhCH}=)], 8.02\left(1 \mathrm{H}, \mathrm{t} ; \underline{\mathrm{HSO}}_{2}\right), 3.97\left(2 \mathrm{H}, \mathrm{d} ; \mathrm{C}_{2} \mathrm{NH}\right)$ and $7.28\left(5 \mathrm{H}, \mathrm{s} ; \underline{\mathrm{PhCH}_{2}}\right)$.

$N$-(N'-Phenylbenzenesulfonamido)-3-carboxy-4-methyl-4-phenyl-3-butenamide (21)

White crystals, m.p. $162^{\circ} \mathrm{C}, 60 \%$ yield in m.w, and $20 \%$ in thermal. FTIR $(\mathrm{KBr}): v\left(\mathrm{~cm}^{-1}\right)=3300-3250(2 \mathrm{NH}), 3400-2400(\mathrm{OH}$; acid $), 1690(\mathrm{CO}$; acid $), 1688$ (CO; amide), 1337 and $1157\left(\mathrm{SO}_{2}\right.$; asy and sym.). $\mathrm{MS}: \mathrm{m} / \mathrm{z}=450\left(\mathrm{M}^{+}, 0 \%\right.$, $\left.\mathrm{C}_{24} \mathrm{H}_{22} \mathrm{~N}_{2} \mathrm{O}_{5} \mathrm{~S}\right), 433\left(40, \mathrm{C}_{24} \mathrm{H}_{21} \mathrm{~N}_{2} \mathrm{O}_{4} \mathrm{~S}\right), 432$ (100, $\left.\mathrm{C}_{24} \mathrm{H}_{20} \mathrm{~N}_{2} \mathrm{O}_{4} \mathrm{~S}\right), 340$ (37.3, Egypt. J. Chem. 53, No. 3 (2010) 
$\left.\mathrm{C}_{18} \mathrm{H}_{14} \mathrm{NO}_{4} \mathrm{~S}\right), \quad 277 \quad\left(0, \quad 4 \quad \mathrm{C}_{18} \mathrm{H}_{15} \mathrm{NO}_{2}\right), \quad 276 \quad\left(62.7, \mathrm{C}_{18} \mathrm{H}_{14} \mathrm{NO}_{2}\right), \quad 248(30$, $\left.\mathrm{C}_{12} \mathrm{H}_{12} \mathrm{~N}_{2} \mathrm{O}_{2} \mathrm{~S}\right), 156\left(28, \mathrm{C}_{6} \mathrm{H}_{6} \mathrm{NO}_{2} \mathrm{~S}\right), 130\left(23.2, \mathrm{C}_{10} \mathrm{H}_{10}\right), 115\left(28.4, \mathrm{C}_{9} \mathrm{H}_{7}\right)$ and 92 $\left(40.4, \mathrm{C}_{6} \mathrm{H}_{6} \mathrm{~N}\right) .{ }^{1} \mathrm{HNMR}\left(\mathrm{DMSO}_{6}\right): \delta(\mathrm{ppm})=7.66(5 \mathrm{H}, \mathrm{s} ; \mathrm{Ph}), 2.36(3 \mathrm{H}, \mathrm{s}$; $\left.\mathrm{PhC}\left(\mathrm{CH}_{3}\right)=\mathrm{C}\right), 3.19(2 \mathrm{H}, \mathrm{s} ; \mathrm{CH} 2 \mathrm{CO}), 10.39\left(1 \mathrm{H}, \mathrm{s} ; \mathrm{NHSO}_{2}\right), 7.36(4 \mathrm{H}, \mathrm{s} ;$ $\left.\mathrm{NPhSO}_{2}\right), 10.16(1 \mathrm{H}, \mathrm{s} ; \mathrm{NHCO}), 7.05-7.23(5 \mathrm{H}, \mathrm{m} ; \mathrm{NHPh})$ and $12.56(1 \mathrm{H}$, broad; $\mathrm{COOH})$.

$N$-( $N$ '-Phenylbenzenesulfonamido)-3-phenylethylidinepyrrolidine-2, 5-dione (22)

Pale yellow crystals, m.p. $136^{\circ} \mathrm{C}, 20 \%$ yield in m.w, and $0 \%$ in thermal. FTIR $(\mathrm{KBr}): v\left(\mathrm{~cm}^{-1}\right)=3248.3(\mathrm{NH}), 1766-1707$ (2CO; imide), 1377 and $1159\left(\mathrm{SO}_{2}\right.$; asy. and sym.). MS: $\mathrm{m} / \mathrm{z}=432\left(\mathrm{M}^{+}, 100 \%, \mathrm{C}_{24} \mathrm{H}_{20} \mathrm{~N}_{2} \mathrm{O}_{4} \mathrm{~S}\right), 340\left(47, \mathrm{C}_{18} \mathrm{H}_{14} \mathrm{NO}_{4} \mathrm{~S}\right)$, $276\left(93.8, \mathrm{C}_{18} \mathrm{H}_{14} \mathrm{NO}_{2}\right), 248\left(29.6, \mathrm{C}_{12} \mathrm{H}_{12} \mathrm{~N}_{2} \mathrm{O}_{2} \mathrm{~S}\right), 233\left(10, \mathrm{C}_{12} \mathrm{H}_{11} \mathrm{NO}_{2} \mathrm{~S}\right), 130$ (29, $\left.\mathrm{C}_{10} \mathrm{H}_{10}\right), 129\left(43.8, \mathrm{C}_{10} \mathrm{H}_{9}\right), 115\left(32.4, \mathrm{C}_{9} \mathrm{H}_{7}\right)$ and $92\left(44, \mathrm{C}_{6} \mathrm{H}_{6} \mathrm{~N}\right),{ }^{1} \mathrm{HNMR}$ $\left(\mathrm{DMSO}_{\mathrm{d}}\right): \delta(\mathrm{ppm})=7.52-7.66(3 \mathrm{H}, \mathrm{m} ; \mathrm{Ph}), 7.89-7.93(2 \mathrm{H}, \mathrm{m} ; \mathrm{Ph}), 2.34(3 \mathrm{H}$, s; $\left.\mathrm{PhC}\left(\mathrm{CH}_{3}\right)=\mathrm{C}\right), 2.63\left(2 \mathrm{H}, \mathrm{s} ; \mathrm{C}_{2} \mathrm{CO}\right), 7.44\left(4 \mathrm{H}, \mathrm{s} ; \mathrm{NPhSO}_{2}\right), 10.44(1 \mathrm{H}, \mathrm{s}$; $\mathrm{NHSO}_{2}$ ) and $7.05-7.32(5 \mathrm{H}, \mathrm{m} ; \mathrm{Ph})$.

$N$-[N'-(4-Methylphenyl)benzenesulfonamido]-3-carboxy-4-methyl-4-phenyl-3butenamide (23)

White crystals, m.p. $210^{\circ} \mathrm{C}, 67 \%$ yield in m.w, and $22 \%$ in thermal. FTIR $(\mathrm{KBr}): v\left(\mathrm{~cm}^{-1}\right)=3300-3255(2 \mathrm{NH}), 3400-2400(\mathrm{OH} ;$ acid $), 1705(\mathrm{CO}$; acid $)$, 1688.5 (CO; amide), 1331 and $1157\left(\mathrm{SO}_{2}\right.$; asy. and sym.). $\mathrm{MS}: \mathrm{m} / \mathrm{z}=464\left(\mathrm{M}^{+}\right.$, $\left.0 \%, \mathrm{C}_{25} \mathrm{H}_{24} \mathrm{~N}_{2} \mathrm{O}_{5} \mathrm{~S}\right), 447\left(80.7, \mathrm{C}_{25} \mathrm{H}_{23} \mathrm{~N}_{2} \mathrm{O}_{4} \mathrm{~S}\right), 446\left(79, \mathrm{C}_{25} \mathrm{H}_{22} \mathrm{~N}_{2} \mathrm{O}_{4} \mathrm{~S}\right), 277$ (15.3, $\left.\mathrm{C}_{18} \mathrm{H}_{15} \mathrm{NO}_{2}\right), 276\left(33, \mathrm{C}_{18} \mathrm{H}_{14} \mathrm{NO}_{2}\right), 130\left(8, \mathrm{C}_{10} \mathrm{H}_{10}\right), 115\left(22.5, \mathrm{C}_{9} \mathrm{H}_{7}\right), 107$ (6.5, $\left.\mathrm{C}_{7} \mathrm{H}_{9} \mathrm{~N}\right), 106\left(100, \mathrm{C}_{7} \mathrm{H}_{8} \mathrm{~N}\right)$ and $91\left(2.2, \mathrm{C}_{7} \mathrm{H}_{7}\right) .{ }^{1} \mathrm{HNMR}\left(\right.$ DMSO-d $\left.\mathrm{d}_{6}\right): \delta(\mathrm{ppm})=$ $7.63(5 \mathrm{H}, \mathrm{s} ; \mathrm{Ph}), 2.35\left(3 \mathrm{H}, \mathrm{s} ; \mathrm{PhC}\left(\mathrm{CH}_{3}\right)=\mathrm{C}\right), 3.18\left(2 \mathrm{H}, \mathrm{s} ; \mathrm{CH}_{2} \mathrm{CO}\right), 10.24(1 \mathrm{H}, \mathrm{s}$;

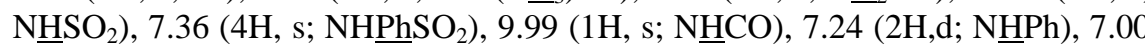
$\left(2 \overline{\mathrm{H}}, \mathrm{d} ; \mathrm{CH}_{3} \underline{\mathrm{Ph}}\right)$ and $2.18\left(\overline{3 \mathrm{H}}, \mathrm{s} ; \mathrm{C}_{3} \mathrm{Ph}\right)$.

$N$-[N'-(4-Methylphenyl)benzenesulfonamido]-3-phenylethylidinepyrrolidine2,5-dione (24)

Yellow crystals, m.p. $130^{\circ} \mathrm{C}, 25 \%$ yield in m.w, and $0 \%$ in thermal. FTIR $(\mathrm{KBr}): v\left(\mathrm{~cm}^{-1}\right)=3250(\mathrm{NH}), 1767-1705\left(2 \mathrm{CO}\right.$; imide), 1378 and $1158\left(\mathrm{SO}_{2}\right.$; asy. and sym.). MS: $\mathrm{m} / \mathrm{z}=446\left(\mathrm{M}^{+}, 90.8 \%, \mathrm{C}_{25} \mathrm{H}_{22} \mathrm{~N}_{2} \mathrm{O}_{4} \mathrm{~S}\right), 277\left(20, \mathrm{C}_{18} \mathrm{H}_{15} \mathrm{NO}_{2}\right), 276$ (28.5, $\left.\mathrm{C}_{18} \mathrm{H}_{14} \mathrm{NO}_{2}\right), 340\left(9.8, \mathrm{C}_{18} \mathrm{H}_{14} \mathrm{NO}_{4} \mathrm{~S}\right), 130\left(12, \mathrm{C}_{10} \mathrm{H}_{10}\right), 115\left(23, \mathrm{C}_{9} \mathrm{H}_{7}\right), 107$ $\left(13, \mathrm{C}_{7} \mathrm{H}_{9} \mathrm{~N}\right), 106\left(100, \mathrm{C}_{7} \mathrm{H}_{8} \mathrm{~N}\right), 102\left(3, \mathrm{C}_{8} \mathrm{H}_{6}\right)$ and $79\left(30, \mathrm{HNO}_{2} \mathrm{~S}\right) .{ }^{1} \mathrm{HNMR}$ $\left(\right.$ DMSO $\left._{6}\right): \delta(\mathrm{ppm})=7.49-7.64(3 \mathrm{H}, \mathrm{m} ; \mathrm{Ph}), 7.78-7.89(2 \mathrm{H}, \mathrm{m} ; \mathrm{Ph}), 2.36(3 \mathrm{H}$, $\left.\mathrm{s} ; \mathrm{PhC}\left(\mathrm{CH}_{3}\right)=\mathrm{C}\right), 2.6\left(2 \mathrm{H}, \mathrm{s} ; \mathrm{CH}_{2} \mathrm{CO}\right), 7.44\left(4 \mathrm{H}, \mathrm{s} ; \mathrm{NPhSO}_{2}\right), 10.28(1 \mathrm{H}, \mathrm{s}$; $\left.\mathrm{NHSO}_{2}\right), 7.00\left[4 \mathrm{H}, \mathrm{s} ; \mathrm{NHPhCH}_{3}\right)$, and $2.13\left(3 \mathrm{H}, \mathrm{s} ; \underline{\mathrm{CH}}_{3} \mathrm{Ph}\right)$.

$N$-[N'-(4-Methoxyphenyl) benzenesulfonamido]-3-carboxy-4-methyl-4-phenyl- 3butenamide (25)

Pale yellow crystals, m.p. $130^{\circ} \mathrm{C}, 52 \%$ yield in m.w, and $72 \%$ in thermal. FTIR (KBr): $v\left(\mathrm{~cm}^{-1}\right)=3300-3243(2 \mathrm{NH}), 3400-2400(\mathrm{OH}$; acid), $1705(\mathrm{CO}$; acid), 1665.5 (CO; amide), 1333 and $1158\left(\mathrm{SO}_{2}\right.$; asy. and sym.). $\mathrm{MS}: \mathrm{m} / \mathrm{z}=480\left(\mathrm{M}^{+}\right.$, 2.3\%, $\left.\mathrm{C}_{25} \mathrm{H}_{24} \mathrm{~N}_{2} \mathrm{O}_{6} \mathrm{~S}\right), 278$ (33, $\left.\mathrm{C}_{13} \mathrm{H}_{13} \mathrm{~N}_{2} \mathrm{O}_{3} \mathrm{~S}\right), 156$ (4.3, $\left.\mathrm{C}_{6} \mathrm{H}_{6} \mathrm{NO}\right), 123$ (20.4, Egypt. J. Chem. 53, No. 3 (2010) 
$\left.\mathrm{C}_{7} \mathrm{H}_{9} \mathrm{NO}\right), 131\left(1, \mathrm{C}_{10} \mathrm{H}_{11}\right), 122\left(100, \mathrm{C}_{6} \mathrm{H}_{4} \mathrm{NS}\right.$ or $\left.\mathrm{C}_{7} \mathrm{H}_{8} \mathrm{NO}\right), 108\left(9, \mathrm{C}_{7} \mathrm{H}_{8} \mathrm{O}\right), 92$ $\left(10.3, \mathrm{C}_{6} \mathrm{H}_{6} \mathrm{~N}\right)$ and $79\left(4, \mathrm{HNO}_{2} \mathrm{~S}\right) .{ }^{1} \mathrm{HNMR}\left(\mathrm{DMSO}-\mathrm{d}_{6}\right): \delta(\mathrm{ppm})=7.61(5 \mathrm{H}, \mathrm{s}$; $\mathrm{Ph}), 2.26\left(3 \mathrm{H}, \mathrm{s} ; \mathrm{PhC}\left(\mathrm{CH}_{3}\right)=\mathrm{C}\right), 3.17(2 \mathrm{H}, \mathrm{s} ; \mathrm{CH} 2 \mathrm{CO}), 10.21\left(1 \mathrm{H}, \mathrm{s} ; \mathrm{NHSO}_{2}\right)$, $\left.7.36(4 \mathrm{H}, \mathrm{s} ; \mathrm{NHPhSO})_{2}\right), 9.78(1 \mathrm{H}, \mathrm{s} ; \mathrm{NHCO}), 6.94(2 \mathrm{H}, \mathrm{d} ; \underline{\mathrm{PhNH}}), 6.81(2 \mathrm{H}, \mathrm{d}$;

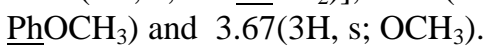

$N$-[N'- (4-Methoxyphenyl) benzenesulfonamido]-3- phenylethylidinepyrrolidine 2,5-dione (26)

Yellow crystals, m.p. $178^{\circ} \mathrm{C}, 40 \%$ yield in m.w, and $0 \%$ in thermal. FTIR $(\mathrm{KBr}): v\left(\mathrm{~cm}^{-1}\right)=3254(\mathrm{NH}), 1767-1705$ (2CO; imide), 1376 and $1160\left(\mathrm{SO}_{2}\right.$; asy. and sym.). $\mathrm{MS}: \mathrm{m} / \mathrm{z}=462\left(\mathrm{M}^{+}, 35 \%, \mathrm{C}_{25} \mathrm{H}_{22} \mathrm{~N}_{2} \mathrm{O}_{5} \mathrm{~S}\right), 130\left(4, \mathrm{C}_{10} \mathrm{H}_{10}\right), 129$ (5.1, $\left.\mathrm{C}_{10} \mathrm{H}_{9}\right), 123\left(8.1, \mathrm{C}_{7} \mathrm{H}_{9} \mathrm{NO}\right), 122\left(100, \mathrm{C}_{7} \mathrm{H}_{8} \mathrm{O}\right.$ or $\left.\mathrm{C}_{6} \mathrm{H}_{4} \mathrm{NS}\right), 115\left(8, \mathrm{C}_{9} \mathrm{H}_{7}\right), 102$ (2, $\left.\mathrm{C}_{8} \mathrm{H}_{6}\right)$ and $96\left(1.9, \mathrm{C}_{4} \mathrm{H}_{2} \mathrm{NO}_{2}\right) .{ }^{1} \mathrm{HNMR}\left(\mathrm{DMSO}-\mathrm{d}_{6}\right): \delta(\mathrm{ppm})=7.33-7.55(3 \mathrm{H}, \mathrm{m}$; $\mathrm{Ph}), 7.80-7.85(2 \mathrm{H}, \mathrm{m} ; \mathrm{Ph}), 2.33\left(3 \mathrm{H}, \mathrm{s} ; \mathrm{PhC}\left(\mathrm{CH}_{3}\right)=\mathrm{C}\right), 2.62\left(2 \mathrm{H}, \mathrm{s} ; \mathrm{CH}_{2} \mathrm{CO}\right)$, 7.44 (4H, s; $\left.\mathrm{NPhSO}_{2}\right), 10.08$ (1H, s; $\left.\underline{\mathrm{HSO}}_{2}\right), 7.01-7.06$ (2H, d; $\left.\underline{\mathrm{PhNH}}\right), 6.81-6.86$ $\left(2 \mathrm{H}, \mathrm{d} ; \mathrm{PhOCH}_{3}\right)$ and $3.68\left(3 \mathrm{H}, \mathrm{s} ; \mathrm{OC}_{3}\right)$.

$N$-[N'-(4-Chlorophenyl)benzenesulfonamido]-3-carboxy-4-methyl-4-phenyl- 3 butenamide (27)

White crystals, $200^{\circ} \mathrm{C}, 30 \%$ yield in yield in m.w, and $37 \%$ in thermal. FTIR $(\mathrm{KBr}): v\left(\mathrm{~cm}^{-1}\right)=3300-3243(2 \mathrm{NH}), 3400-2400$ (OH; acid), 1705(CO; acid), 1665.5 (CO; amide), 1333 and $1158\left(\mathrm{SO}_{2}\right.$; asy. and sym.). $\mathrm{MS}: \mathrm{m} / \mathrm{z}=484\left(\mathrm{M}^{+}\right.$, 5\%, $\left.\mathrm{C}_{24} \mathrm{H}_{21} \mathrm{~N}_{2} \mathrm{O}_{5} \mathrm{SCl}\right) 466\left(100, \mathrm{C}_{24} \mathrm{H}_{19} \mathrm{~N}_{2} \mathrm{O}_{4} \mathrm{SCl}\right), 340$ (55.5, $\left.\mathrm{C}_{18} \mathrm{H}_{14} \mathrm{NO}_{4} \mathrm{~S}\right), 277$ $\left(19, \mathrm{C}_{18} \mathrm{H}_{15} \mathrm{NO}_{2}\right), \quad 276\left(99.4, \mathrm{C}_{18} \mathrm{H}_{14} \mathrm{NO}_{2}\right), 220\left(6.6, \mathrm{C}_{16} \mathrm{H}_{14} \mathrm{~N}\right), 157$ (9.6, $\left.\mathrm{C}_{11} \mathrm{H}_{9} \mathrm{O}\right), 126\left(46, \mathrm{C}_{6} \mathrm{H}_{5} \mathrm{NCl}\right), 129\left(25.6, \mathrm{C}_{10} \mathrm{H}_{9}\right), 115\left(18.8, \mathrm{C}_{9} \mathrm{H}_{7}\right)$ and $79(2$, $\left.\mathrm{HNO}_{2} \mathrm{~S}\right)$.

$N$-[N'-(4-Chlorophenyl) benzenesulfonamido]-3-phenylethylidinepyrrolidine-2, 5-dione (28)

Pale yellow crystals, m.p. $110^{\circ} \mathrm{C}, 63 \%$ yield in m.w, and $37 \%$ in thermal. FTIR (KBr): $v\left(\mathrm{~cm}^{-1}\right)=3241(\mathrm{NH}), 1766-1706$ (2CO; imide), 1375and 1160 $\left(\mathrm{SO}_{2}\right.$; asy. and sym.). MS: $\mathrm{m} / \mathrm{z}=466\left(\mathrm{M}^{+}, 100 \%, \mathrm{C}_{24} \mathrm{H}_{19} \mathrm{~N}_{2} \mathrm{O}_{4} \mathrm{SCl}\right), 340$ (55.5, $\left.\mathrm{C}_{18} \mathrm{H}_{14} \mathrm{NO}_{4} \mathrm{~S}\right), 277\left(19, \mathrm{C}_{18} \mathrm{H}_{15} \mathrm{NO}_{2}\right), \quad 276\left(99.4, \mathrm{C}_{18} \mathrm{H}_{14} \mathrm{NO}_{2}\right), 220\left(6.6, \mathrm{C}_{16} \mathrm{H}_{14} \mathrm{~N}\right)$, $157\left(9.6, \mathrm{C}_{11} \mathrm{H}_{9} \mathrm{O}\right), 126\left(46, \mathrm{C}_{6} \mathrm{H}_{5} \mathrm{NCl}\right), 129\left(25.6, \mathrm{C}_{10} \mathrm{H}_{9}\right), 115\left(18.8, \mathrm{C}_{9} \mathrm{H}_{7}\right)$ and $79\left(2, \mathrm{HNO}_{2} \mathrm{~S}\right)$.

$N$-[N'-(4-Nitrophenyl) benzenesulfonamido]-3-carboxy-4-methyl-4-phenyl-3butenamide (29)

Pale yellow crystals, m.p. $119^{\circ} \mathrm{C}, 79 \%$ yield in m.w, and $61 \%$ in thermal. FTIR (KBr): $v\left(\mathrm{~cm}^{-1}\right)=3360-3355(2 \mathrm{NH}), 3400-2400(\mathrm{OH} ;$ acid $), 1700(\mathrm{CO}$; acid), 1690 (CO; amide), 1344 and $1160\left(\mathrm{SO}_{2}\right.$; asy. and sym.). MS: $\mathrm{m} / \mathrm{z}=495$ $\left(\mathrm{M}^{+}, 1.8 \%, \mathrm{C}_{24} \mathrm{H}_{21} \mathrm{~N}_{3} \mathrm{O}_{7} \mathrm{~S}\right), 294\left(4, \mathrm{C}_{18} \mathrm{H}_{17} \mathrm{NO}_{3}\right), 293\left(19, \mathrm{C}_{12} \mathrm{H}_{11} \mathrm{~N}_{3} \mathrm{O}_{4} \mathrm{~S}\right), 292$ (2.5, $\left.\mathrm{C}_{12} \mathrm{H}_{10} \mathrm{~N}_{3} \mathrm{O}_{4} \mathrm{~S}\right), 202\left(9.2, \mathrm{C}_{12} \mathrm{H}_{10} \mathrm{O}_{3}\right), 156\left(100, \mathrm{C}_{6} \mathrm{H}_{6} \mathrm{NO}_{2} \mathrm{~S}\right), 137\left(0.9, \mathrm{C}_{6} \mathrm{H}_{5} \mathrm{~N}_{2} \mathrm{O}_{2}\right)$, $131\left(3, \mathrm{C}_{10} \mathrm{H}_{11}\right), 130\left(13, \mathrm{C}_{10} \mathrm{H}_{10}\right), 115\left(16.3, \mathrm{C}_{9} \mathrm{H}_{7}\right)$ and $92\left(87, \mathrm{C}_{6} \mathrm{H}_{6} \mathrm{~N}\right)$. 
$N$-[N'-(1-Naphthyl) benzenesulfonamido]-3-carboxy-4-methyl-4-phenyl-3butenamide (30)

Pale violet crystals, m.p. $129^{\circ} \mathrm{C}, 53 \%$ yield in m.w, and $41 \%$ in thermal. FTIR $(\mathrm{KBr}): v\left(\mathrm{~cm}^{-1}\right)=3300-3248(2 \mathrm{NH}), 3400-2400(\mathrm{OH} ; \mathrm{acid}), 1700(\mathrm{CO} ; \mathrm{acid}), 1698$ (CO; amide), 1399.9 and $1159\left(\mathrm{SO}_{2}\right.$; asy. and sym.). $\mathrm{MS}: \mathrm{m} / \mathrm{z}=500\left(\mathrm{M}^{+}, 0 \%\right.$, $\left.\mathrm{C}_{28} \mathrm{H}_{24} \mathrm{~N}_{2} \mathrm{O}_{5} \mathrm{~S}\right), 328 \quad\left(1.3, \mathrm{C}_{17} \mathrm{H}_{16} \mathrm{~N}_{2} \mathrm{O}_{3} \mathrm{~S}\right), \quad 327\left(4, \mathrm{C}_{17} \mathrm{H}_{15} \mathrm{~N}_{2} \mathrm{O}_{3} \mathrm{~S}\right), 298$ (12.7, $\left.\mathrm{C}_{16} \mathrm{H}_{14} \mathrm{~N}_{2} \mathrm{O}_{2} \mathrm{~S}\right), 297\left(2.4, \mathrm{C}_{16} \mathrm{H}_{13} \mathrm{~N}_{2} \mathrm{O}_{2} \mathrm{~S}\right), 143\left(45, \mathrm{C}_{10} \mathrm{H}_{9} \mathrm{~N}\right), 142\left(27.7, \mathrm{C}_{10} \mathrm{H}_{8} \mathrm{~N}\right)$, $130\left(65, \mathrm{C}_{10} \mathrm{H}_{10}\right), 129\left(92, \mathrm{C}_{10} \mathrm{H}_{9}\right), 115\left(100, \mathrm{C}_{9} \mathrm{H}_{7}\right)$ and $79\left(4, \mathrm{HNO}_{2} \mathrm{~S}\right) .{ }^{1} \mathrm{HNMR}$ $\left(\right.$ DMSO-d $\left._{6}\right): \delta(\mathrm{ppm})=7.75-7.87(3 \mathrm{H}, \mathrm{m} ; \mathrm{Ph}), 8.07(2 \mathrm{H}, \mathrm{m} ; \mathrm{Ph}), 2.36(3 \mathrm{H}, \mathrm{s}$; $\left.\mathrm{PhC}\left(\mathrm{CH}_{3}\right)=\mathrm{C}\right), 3.19 \quad\left(2 \mathrm{H}, \mathrm{s} ; \mathrm{CH}_{2} \mathrm{CO}\right), 10.49 \quad\left(1 \mathrm{H}, \mathrm{s} ; \mathrm{N}_{\mathrm{HSO}}\right), 7.44(4 \mathrm{H}, \mathrm{s}$; $\left.\mathrm{NH} \underline{\mathrm{PhSO}_{2}}\right), 10.20(1 \mathrm{H}, \mathrm{s} ; \mathrm{N} \underline{\mathrm{HCO}})$ and $7.80-7.10\left(7 \mathrm{H}, \mathrm{m} ; \mathrm{C}_{8} \underline{\mathrm{H}}_{7}\right)$.

$N$-[N'-(1-Naphthyl) benzenesulfonamido]-3-phenylethylidinepyrrolidine-2,5dione (31)

Violet crystals, m.p. $98^{\circ} \mathrm{C}, 15 \%$ yield in m.w, and $0 \%$ in thermal. FTIR $(\mathrm{KBr}): v\left(\mathrm{~cm}^{-1}\right)=3251(\mathrm{NH}), 1766.5-1706(2 \mathrm{CO}$; imide $), 1372$ and $1159\left(\mathrm{SO}_{2}\right.$; asy. and sym.). MS: $\mathrm{m} / \mathrm{z}=482\left(\mathrm{M}^{+}, 0 \%, \mathrm{C}_{28} \mathrm{H}_{22} \mathrm{~N}_{2} \mathrm{O}_{4} \mathrm{~S}\right), 328\left(14.1, \mathrm{C}_{17} \mathrm{H}_{16} \mathrm{~N}_{2} \mathrm{O}_{3} \mathrm{~S}\right)$, $327\left(64.7, \mathrm{C}_{17} \mathrm{H}_{15} \mathrm{~N}_{2} \mathrm{O}_{3} \mathrm{~S}\right), 298\left(13.5, \mathrm{C}_{16} \mathrm{H}_{14} \mathrm{~N}_{2} \mathrm{O}_{2} \mathrm{~S}\right), 297\left(4, \mathrm{C}_{16} \mathrm{H}_{13} \mathrm{~N}_{2} \mathrm{O}_{2} \mathrm{~S}\right)$, 202(23.8, $\left.\mathrm{C}_{12} \mathrm{H}_{10} \mathrm{O}_{3}\right), 143\left(13, \mathrm{C}_{10} \mathrm{H}_{9} \mathrm{~N}\right), 142\left(11.4, \mathrm{C}_{10} \mathrm{H}_{8}\right), 130\left(90.3, \mathrm{C}_{10} \mathrm{H}_{10}\right)$, $129\left(100, \mathrm{C}_{10} \mathrm{H}_{9}\right)$ and $115\left(88, \mathrm{C}_{9} \mathrm{H}_{7}\right) .{ }^{1} \mathrm{HNMR}\left(\mathrm{DMSO}_{-} \mathrm{d}_{6}\right): \delta(\mathrm{ppm})=7.80-7.88$ $(3 \mathrm{H}, \mathrm{m} ; \mathrm{Ph}), 8.07(2 \mathrm{H}, \mathrm{m} ; \mathrm{Ph}), 2.34\left(3 \mathrm{H}, \mathrm{s} ; \mathrm{PhC}\left(\mathrm{CH}_{3}\right)=\mathrm{C}\right), 3.01\left(2 \mathrm{H}, \mathrm{s} ; \mathrm{CH}_{2} \mathrm{CO}\right)$, $7.45\left(4 \mathrm{H}, \mathrm{s} ; \mathrm{NPhSO}_{2}\right), 10.40\left(1 \mathrm{H}, \mathrm{s} ; \mathrm{NHSO}_{2}\right)$ and 7.17-7.62 (7H, m; $\left.\mathrm{C}_{8} \mathrm{H}_{7}\right)$.

$N$-(N'-Benzylbenzenesulfonamido)-3-carboxy-4-methyl-4-phenyl-3-butenamide (32)

White crystals, m.p. $153^{\circ} \mathrm{C}, 85 \%$ yield in m.w, and $57 \%$ in thermal. FTIR $(\mathrm{KBr}): v\left(\mathrm{~cm}^{-1}\right)=3321-3267(2 \mathrm{NH}), 3400-2400(\mathrm{OH} ; \mathrm{acid}), 1700(\mathrm{CO}$; acid $), 1680$ (CO; amide), 1328 and 1157( $\mathrm{SO}_{2}$; asy. and sym.). $\mathrm{MS}: \mathrm{m} / \mathrm{z}=464\left(\mathrm{M}^{+}, 0 \%\right.$, $\left.\mathrm{C}_{25} \mathrm{H}_{24} \mathrm{~N}_{2} \mathrm{O}_{5} \mathrm{~S}\right), 446\left(22.4, \mathrm{C}_{25} \mathrm{H}_{22} \mathrm{~N}_{2} \mathrm{O}_{4} \mathrm{~S}\right), 262$ (32, $\left.\mathrm{C}_{13} \mathrm{H}_{14} \mathrm{~N}_{2} \mathrm{O}_{2} \mathrm{~S}\right), 202$ (36.5, $\left.\mathrm{C}_{12} \mathrm{H}_{10} \mathrm{O}_{3}\right), 157\left(9.2, \mathrm{C}_{11} \mathrm{H}_{9} \mathrm{O}\right), 156\left(15.2, \mathrm{C}_{6} \mathrm{H}_{6} \mathrm{NO}_{2} \mathrm{~S}\right), 130\left(41.4, \mathrm{C}_{10} \mathrm{H}_{10}\right), 129$ (74, $\left.\mathrm{C}_{10} \mathrm{H}_{9}\right), 115\left(46, \mathrm{C}_{9} \mathrm{H}_{7}\right), 106\left(100, \mathrm{C}_{7} \mathrm{H}_{8} \mathrm{~N}\right), 92\left(66.4, \mathrm{C}_{6} \mathrm{H}_{6} \mathrm{~N}\right)$ and $79\left(14, \mathrm{HNO}_{2} \mathrm{~S}\right)$.

$N$-(4-Methylphenyl)-3-carboxy-4-methyl-4-phenyl-3-butenamide (33)

White crystals, m.p. $162^{\circ} \mathrm{C}, 0 \%$ yield in m.w, and $26 \%$ in thermal. FTIR $(\mathrm{KBr}): v\left(\mathrm{~cm}^{-1}\right)=3300(\mathrm{NH}), 3100-2500(\mathrm{OH}$ acid$), 1680(\mathrm{CO}$, acid$)$, and 1650 (CO, amide). MS: m/z = $309\left(\mathrm{M}^{+}, 25 \%, \mathrm{C}_{19} \mathrm{H}_{19} \mathrm{NO}_{3}\right), 291\left(22, \mathrm{C}_{19} \mathrm{H}_{17} \mathrm{NO}_{2}\right), 202$ $\left(30, \mathrm{C}_{12} \mathrm{H}_{10} \mathrm{O}_{3}\right), 130\left(25, \mathrm{C}_{10} \mathrm{H}_{10}\right), 129\left(33, \mathrm{C}_{10} \mathrm{H}_{9}\right)$, and $107\left(100, \mathrm{C}_{7} \mathrm{H}_{9} \mathrm{~N}\right)$.

$N$-(4-Methylyphenyl)-3-phenylethylidinepyrrolidine-2, 5-dione (34)

Pale brown crystals, m.p. $118^{\circ} \mathrm{C}, 93 \%$ yield in m.w, and $0 \%$ in thermal. FTIR $(\mathrm{KBr}): \mathrm{v}\left(\mathrm{cm}^{-1}\right)=1756.8-1699\left(2 \mathrm{CO}\right.$; imide). $\mathrm{MS}: \mathrm{m} / \mathrm{z}=307\left(\mathrm{M}^{+}, 100 \%\right.$, $\mathrm{C}_{19} \mathrm{H}_{17} \mathrm{NO}_{3}$ ), $292\left(2.6, \mathrm{C}_{18} \mathrm{H}_{14} \mathrm{NO}_{3}\right), 202\left(1.7, \mathrm{C}_{12} \mathrm{H}_{12} \mathrm{NO}_{2}\right), 149\left(33, \mathrm{C}_{8} \mathrm{H}_{7} \mathrm{NO}_{2}\right)$, $130\left(91.2, \mathrm{C}_{10} \mathrm{H}_{10}\right), 129\left(75, \mathrm{C}_{10} \mathrm{H}_{9}\right), 115\left(53, \mathrm{C}_{9} \mathrm{H}_{7}\right)$ and $108\left(16, \mathrm{C}_{7} \mathrm{H}_{8} \mathrm{O}\right)$.

$N$-(4-Methoxyphenyl)-3-carboxy-4-methyl-4-phenyl-3-butenamide (35)

Gray crystals, m.p. $172^{\circ} \mathrm{C}, 0 \%$ yield in m.w, and $45 \%$ in thermal. FTIR $(\mathrm{KBr}): v\left(\mathrm{~cm}^{-1}\right)=3298(\mathrm{NH}), 3100-2500(\mathrm{OH}$ acid$), 1698(\mathrm{CO}$, acid$)$, and 1663 
(CO, amide). MS: $\mathrm{m} / \mathrm{z}=325\left(\mathrm{M}^{+}, 8 \%, \mathrm{C}_{19} \mathrm{H}_{19} \mathrm{NO}_{4}\right), 307 \quad\left(8 \%, \mathrm{C}_{19} \mathrm{H}_{17} \mathrm{NO}_{3}\right), 202$ $\left(13 \%, \mathrm{C}_{12} \mathrm{H}_{10} \mathrm{O}_{3}\right), 130\left(31 \%, \mathrm{C}_{10} \mathrm{H}_{10}\right), 129\left(53 \%, \mathrm{C}_{10} \mathrm{H}_{9}\right)$, and $123(100 \%$, $\left.\mathrm{C}_{7} \mathrm{H}_{9} \mathrm{NO}\right) .{ }^{1} \mathrm{HNMR}\left(\mathrm{DMSO}-\mathrm{d}_{6}\right): \delta(\mathrm{ppm})=7.31-7.43(5 \mathrm{H}, \mathrm{m} ; \mathrm{Ph}), 2.33(3 \mathrm{H}, \mathrm{s}$; $\left.\mathrm{PhC}\left(\mathrm{CH}_{3}\right)=\mathrm{C}\right), 3.13\left(2 \mathrm{H}, \mathrm{s} ; \mathrm{CH}_{2} \mathrm{CO}\right), 9.57(1 \mathrm{H}, \mathrm{s} ; \mathrm{NHCO}), 7.23-7.25(2 \mathrm{H}, \mathrm{d}$; $\mathrm{NHPh}), 6.82-6.85\left(2 \mathrm{H}, \mathrm{d} ; \underline{\mathrm{PhOCH}}_{3}\right)$, and $3.70\left(3 \mathrm{H}, \mathrm{s} ; \overline{\mathrm{OCH}}_{3}\right)$.

$N$-(4-Methoxyphenyl)-3-phenylethylidinepyrrolidine-2, 5-dione (36)

Gray crystals, m.p. $144^{\circ} \mathrm{C}, 97 \%$ yield in m.w, and $0 \%$ in thermal. FTIR $(\mathrm{KBr}): \mathrm{v}\left(\mathrm{cm}^{-1}\right)=1756.8-1699$ (2CO; imide). $\mathrm{MS}: \mathrm{m} / \mathrm{z}=307\left(\mathrm{M}^{+}, 100 \%\right.$, $\mathrm{C}_{19} \mathrm{H}_{17} \mathrm{NO}_{3}$ ), $292\left(2.6, \mathrm{C}_{18} \mathrm{H}_{14} \mathrm{NO}_{3}\right), 202\left(1.7, \mathrm{C}_{12} \mathrm{H}_{12} \mathrm{NO}_{2}\right), 149\left(33, \mathrm{C}_{8} \mathrm{H}_{7} \mathrm{NO}_{2}\right)$, $130\left(91.2, \mathrm{C}_{10} \mathrm{H}_{10}\right), 129\left(75, \mathrm{C}_{10} \mathrm{H}_{9}\right), 115\left(53, \mathrm{C}_{9} \mathrm{H}_{7}\right)$ and $108\left(16, \mathrm{C}_{7} \mathrm{H}_{8} \mathrm{O}\right)$.

$N$-(4-Chlorophenyl)-3-carboxy-4-methyl-4-phenyl-3-butenamide (37)

White crystals, m.p. $176^{\circ} \mathrm{C}, 0 \%$ yield in m.w, and $49 \%$ in thermal. FTIR (KBr): $v\left(\mathrm{~cm}^{-1}\right)=3290(\mathrm{NH}), 3100-2500(\mathrm{OH} ; \mathrm{acid}), 1695(\mathrm{CO} ;$ acid $)$ and $1660(\mathrm{CO}$; amid). MS: $\mathrm{m} / \mathrm{z}=329\left(\mathrm{M}^{+}, 4 \%, \mathrm{C}_{18} \mathrm{H}_{16} \mathrm{NO}_{3} \mathrm{Cl}\right), 311\left(6 \%, \mathrm{C}_{18} \mathrm{H}_{14} \mathrm{NO}_{2} \mathrm{Cl}\right), 202(9 \%$, $\left.\mathrm{C}_{12} \mathrm{H}_{10} \mathrm{O}_{3}\right), 130\left(27 \%, \mathrm{C}_{10} \mathrm{H}_{10}\right), 129\left(64 \%, \mathrm{C}_{10} \mathrm{H}_{9}\right)$, and $127\left(100 \% \mathrm{C}_{6} \mathrm{H}_{6} \mathrm{NCl}\right)$.

$\mathrm{N}$-(4-Chlorophenyl)-3-phenylethylidinepyrrolidine-2, 5-dione (38)

Brown crystals, m.p. $146^{\circ} \mathrm{C}, 80 \%$ yield in m.w, and $0 \%$ in thermal. FTIR $(\mathrm{KBr}): \mathrm{v}\left(\mathrm{cm}^{-1}\right)=1755-1704(2 \mathrm{CO} ;$ imide $) . \mathrm{MS}: \mathrm{m} / \mathrm{z}=311\left(\mathrm{M}^{+}, 68.7 \%\right.$, $\left.\mathrm{C}_{18} \mathrm{H}_{14} \mathrm{NO}_{2} \mathrm{Cl}\right), 158\left(9.2, \mathrm{C}_{11} \mathrm{H}_{10} \mathrm{O}\right), 131\left(12.5, \mathrm{C}_{10} \mathrm{H}_{11}\right), 130\left(100, \mathrm{C}_{10} \mathrm{H}_{10}\right), 129$ $\left(83.2, \mathrm{C}_{10} \mathrm{H}_{9}\right), 127\left(19, \mathrm{C}_{6} \mathrm{H}_{6} \mathrm{NCl}\right), 125\left(7.8, \mathrm{C}_{6} \mathrm{H}_{4} \mathrm{NCl}\right), 115\left(70.2, \mathrm{C}_{9} \mathrm{H}_{7}\right), 111$ $\left(5.3, \mathrm{C}_{6} \mathrm{H}_{4} \mathrm{Cl}\right)$ and $102\left(8, \mathrm{C}_{8} \mathrm{H}_{6}\right)$.

N-(4-Nitrophenyl)-3-carboxy-4-methyl-4-phenyl-3-butenamide (39)

Yellow crystals, m.p. $182^{\circ} \mathrm{C}, 0 \%$ yield in m.w, and $42 \%$ in thermal. FTIR $(\mathrm{KBr}): v\left(\mathrm{~cm}^{-1}\right)=3291(\mathrm{NH}), 3100-2500(\mathrm{OH}$; acid $), 1684(\mathrm{CO}$; acid $)$ and 1640 (CO; amid). MS: $\mathrm{m} / \mathrm{z}=340\left(\mathrm{M}^{+}, 0 \%, \mathrm{C}_{18} \mathrm{H}_{16} \mathrm{~N}_{2} \mathrm{O}_{5}\right), 322\left(29 \%, \mathrm{C}_{18} \mathrm{H}_{14} \mathrm{~N}_{2} \mathrm{O}_{4}\right), 202$ $\left(21 \%, \mathrm{C}_{12} \mathrm{H}_{10} \mathrm{O}_{3}\right), 138\left(54 \%, \mathrm{C}_{6} \mathrm{H}_{6} \mathrm{~N}_{2} \mathrm{O}_{2}\right)$, and $129\left(100 \%, \mathrm{C}_{10} \mathrm{H}_{9}\right)$

$N$-(4-Nitrophenyl)-3-phenylethylidinepyrrolidine-2,5-dione (40)

Brown crystals, m.p. $150^{\circ} \mathrm{C}, 89 \%$ yield in m.w, and $0 \%$ in thermal. FTIR $(\mathrm{KBr}): \mathrm{v}\left(\mathrm{cm}^{-1}\right)=1754.4-1703$ (2CO; imide). $\mathrm{MS}: \mathrm{m} / \mathrm{z}=322\left(\mathrm{M}^{+}, 5 \%\right.$, $\left.\mathrm{C}_{18} \mathrm{H}_{14} \mathrm{~N}_{2} \mathrm{O}_{4}\right), 202\left(7, \mathrm{C}_{12} \mathrm{H}_{12} \mathrm{NO}_{2}\right), 158\left(6.6, \mathrm{C}_{11} \mathrm{H}_{10} \mathrm{O}\right), 157$ (11.2, $\left.\mathrm{C}_{11} \mathrm{H}_{9} \mathrm{O}\right), 136$ $\left(2.3, \mathrm{C}_{6} \mathrm{H}_{4} \mathrm{~N}_{2} \mathrm{O}_{2}\right), 130\left(100, \mathrm{C}_{10} \mathrm{H}_{10}\right), 129\left(84.6, \mathrm{C}_{10} \mathrm{H}_{9}\right), 122\left(2.3, \mathrm{C}_{6} \mathrm{H}_{4} \mathrm{NO}_{2}\right)$, and $115\left(85, \mathrm{C}_{9} \mathrm{H}_{7}\right)$.

$N$-(1-Naphthyl)-3-carboxy-4-methyl-4-phenyl-3-butenamide (41)

Pale violet crystals, m.p. $180^{\circ} \mathrm{C}, 19 \%$ yield in m.w, and $35 \%$ in thermal. FTIR $(\mathrm{KBr}): v\left(\mathrm{~cm}^{-1}\right)=3270(\mathrm{NH}), 3100-2500(\mathrm{OH}$; acid $), 1700(\mathrm{CO}$; acid $)$ and 1640 (CO; amid). MS: $\mathrm{m} / \mathrm{z}=345\left(\mathrm{M}^{+}, 5 \%, \mathrm{C}_{22} \mathrm{H}_{19} \mathrm{NO}_{3}\right), 327\left(3 \%, \mathrm{C}_{22} \mathrm{H}_{17} \mathrm{NO}_{2}\right), 202$ $\left(5 \%, \mathrm{C}_{12} \mathrm{H}_{10} \mathrm{O}_{3}\right), 143\left(100 \%, \mathrm{C}_{10} \mathrm{H}_{9} \mathrm{~N}\right), 130\left(15 \%, \mathrm{C}_{10} \mathrm{H}_{10}\right)$, and $129\left(27 \%, \mathrm{C}_{10} \mathrm{H}_{9}\right)$

$N$-(1-Naphthyl)-3-phenylethylidinepyrrolidine-2, 5-dione (42)

Dark gray crystals, m.p. $135^{\circ} \mathrm{C}, 71 \%$ yield in m.w, and $0 \%$ in thermal. FTIR $(\mathrm{KBr}): \mathrm{v}\left(\mathrm{cm}^{-1}\right)=1762-1703$ (2CO; imide). $\mathrm{MS}: \mathrm{m} / \mathrm{z}=327\left(\mathrm{M}^{+}, 96.4 \%\right.$, $\mathrm{C}_{22} \mathrm{H}_{17} \mathrm{NO}_{2}$ ), $270\left(4.1, \mathrm{C}_{20} \mathrm{H}_{16} \mathrm{~N}\right), 202\left(2, \mathrm{C}_{12} \mathrm{H}_{12} \mathrm{NO}_{2}\right), 169$ (25.5, $\left.\mathrm{C}_{11} \mathrm{H}_{7} \mathrm{NO}\right), 157$ Egypt. J. Chem. 53, No. 3 (2010) 
(9.5, $\left.\mathrm{C}_{11} \mathrm{H}_{9} \mathrm{O}\right), 158\left(7.6, \mathrm{C}_{11} \mathrm{H}_{10} \mathrm{O}\right), 131\left(7, \mathrm{C}_{10} \mathrm{H}_{11}\right), 130\left(100, \mathrm{C}_{10} \mathrm{H}_{10}\right) 129$ (64, $\left.\mathrm{C}_{10} \mathrm{H}_{9}\right), 128\left(46, \mathrm{C}_{10} \mathrm{H}_{8}\right), 127\left(23, \mathrm{C}_{10} \mathrm{H}_{7}\right)$ and $115\left(53, \mathrm{C}_{9} \mathrm{H}_{7}\right)$.

N-Benzyl-3-carboxy-4-methyl-4-phenyl-3-butenamide (43)

White crystals, m.p. $142^{\circ} \mathrm{C}, 92 \%$ yield in m.w, and $71 \%$ in thermal. FTIR $(\mathrm{KBr}): v\left(\mathrm{~cm}^{-1}\right)=3263(\mathrm{NH}), 3100-2500(\mathrm{OH}$; acid $), 1700(\mathrm{CO}$; acid $)$ and 1640 (CO; amid). MS: $\mathrm{m} / \mathrm{z}=309\left(6 \%, \mathrm{C}_{19} \mathrm{H}_{19} \mathrm{NO}_{3}\right), 291\left(20 \% \mathrm{C}_{19} \mathrm{H}_{17} \mathrm{NO}_{2}\right), 202(23.8$, $\left.\mathrm{C}_{12} \mathrm{H}_{10} \mathrm{O}_{3}\right), 143\left(13, \mathrm{C}_{10} \mathrm{H}_{9} \mathrm{~N}\right), 142\left(11.4, \mathrm{C}_{10} \mathrm{H}_{8}\right), 130\left(90.3, \mathrm{C}_{10} \mathrm{H}_{10}\right), 202$ (9, $\left.\mathrm{C}_{12} \mathrm{H}_{10} \mathrm{O}_{3}\right) 130\left(35, \mathrm{C}_{10} \mathrm{H}_{10}\right), 129\left(46, \mathrm{C}_{10} \mathrm{H}_{9}\right), 107\left(18, \mathrm{C}_{7} \mathrm{H}_{9} \mathrm{~N}\right)$, and $91(100$, $\left.\mathrm{C}_{7} \mathrm{H}_{7}\right) .{ }^{1} \mathrm{HNMR}\left(\mathrm{DMSO}-\mathrm{d}_{6}\right): \delta(\mathrm{ppm})=7.13-7.40(10 \mathrm{H}, \mathrm{m} ; 2 \mathrm{Ph}), 2.2(3 \mathrm{H}, \mathrm{s}$; $\left.\mathrm{C}_{3}\right), 2.9\left(2 \mathrm{H}, \mathrm{s} ; \underline{\mathrm{CH}}_{2} \mathrm{CO}\right), 8.99(1 \mathrm{H}, \mathrm{s} ; \mathrm{N} \underline{\mathrm{HCO}})$, and $4.24-4.26(2 \mathrm{H}, \mathrm{d}$, $\mathrm{C}_{2} \mathrm{NH}$ ).

\section{Conclusion}

- In the present work, the comparison shows that the microwave irradiation furnishes the formation of the products in excellent yields, shorter time, enhances cyclization, significantly improves the reaction products quantitatively, qualitatively, economically, and environmentally (green chemistry), much more than thermal heating. Moreover, microwave irradiation reactions show regiospecific property, more than thermal ones.

- The results obtained from microwave irradiation of $\mathrm{N}$-substituted 4aminobenzenesulfonamides with $\alpha, \beta$-unsaturated anhydrides show low reactivity than the corresponding non-sulfonated amines. This low reactivity can be attributed to the presence of bezenesulfonamido group.

\section{General Remarks}

Microwave irradiation was carried out in a Galanz Microwave Oven, WP1000AP30-2, Chemistry Department, University College of Women for Arts, Science and Education, Ain Shams University. Spectral measurements were carried out at Micro Analytical Centre, Faculty of Science, Cairo University using:

(a) FTIR PERKIN-ELMER-1430 infrared spectrophotometer; IR spectra.

(b) GCMS QP 1000 EX Shimaedzy; MS spectra.

(c) Varian Gemmi (200 MHz); ${ }^{1}$ HNMR spectra. 


\section{References}

1. Gedye, R.N., Smith, F., Westaway, K., Ali, H., Baldisera, L., Laberge, L. and Rousell, The use of microwave ovens for rapid organic synthesis J. Tetrahedron Letters, 27, 279- 282 (1986).

2. Loupy, A., Petit, A., Hamelin, J., Texier-Boullet, F., Taquault, P. and Mathe, D., New Solvent-Free Organic Synthesis, 1213-1234 (1998).

3. Hefny, H.A. and Abdallah, Sh.M., Microwave Enhanced Synthesis of N- Substituted Homophthalimide and o-(N-Substituted Acetamido)Benzoic Acid Derivatives, International J. Chem., 17(4), 279-287 (2007).

4. Abdallah, Shadia M., Hefny Halima A., El Toukhy Afaf M. and Abdelmonem, Fatehya I. Microwave Irradiation Synthesis of Butanedioic Hydrazide, Butenamides and Butanimides, International J. Chem. 20 (1), 11-20 (2009).

5. Pandita, Sh.S., Panditb, V.U. and Bandgarc, B.P., Rapid and efficient synthesis of sulfonamides from sulfonic acid and amines using cyanuric chloride-DMF adduct, Journal of Sulfur Chemistry, Journal of Sulfur Chemistry, 29 (6), 619-622 (2008).

6. Stranix, B.R., Lavalle, J.F., Sevigny, G., Telle, J., Perron, V., Leberre, N., Harbart, D. amd Wu , J.J., Bioorg. Med. Chem. Lett. 6, 3459 (2006).

7. Yang, G.F. and Yang, H.Z., Chin. J. Chem. 17, 650 (1999).

8. El-Wahhab, S., El-Assal, L. and Abd-Elaziz, S., Stobbe, H., Egypt J. Chem. 11, 293 (1968) Stobbe condensation, Ber., 26, 2312 (1893).

9. El-Wahhab, S. and El-Assal, L., D. K. and Bagavant G. Banerjee, J. Ind. Chem. Soc. 46, 927 (1969) The stobbe condensation of anisaldehyde and dimethyl succinate, Proceeding Mathematical Sciences, 50, 282-288 (1959).

10. Vogel A.I., Text Book of Practical Organic Chemistry, fourth edition, 651-2 (1976). 


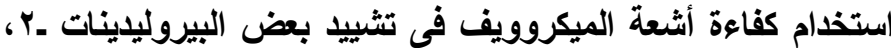

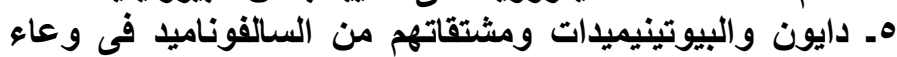 واحد ومقارنتها بالطريقة التقليدية بالتسخين الحرارى}

شادية محمود عبدالله

قسم الكيمياء ـ كلية البنات للآداب و العلوم و التربية ـ جامعة عين شمس ـ القاهرة ـ

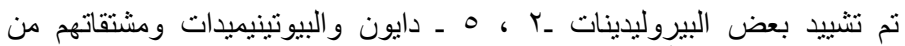

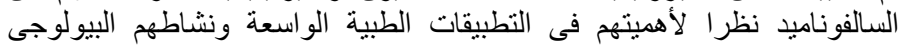
كمبيدات للبكتريا و الفطريات وكمبيدات حشرية ، وذلكاتية بإستخدام طريقة أمنة

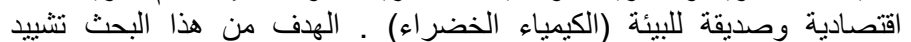

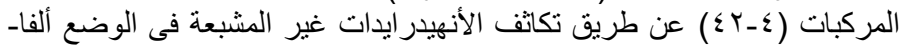

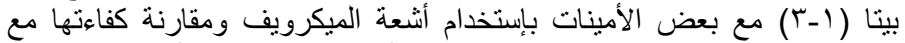

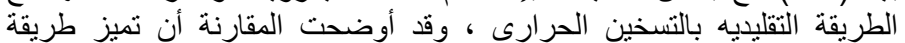

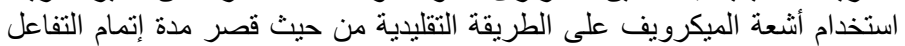

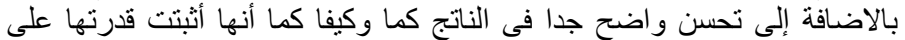

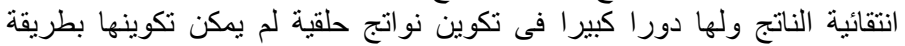
التسخين التقليدية و

وقد أثبتت مقارنة نتائج تكاثف الأنهيدريدات غير المشبعة في الوضع ألفات الفيات ـ بيتا

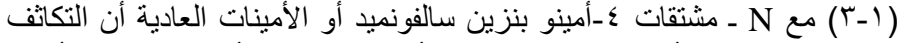

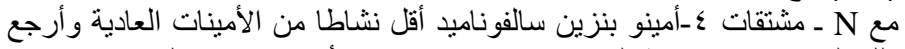

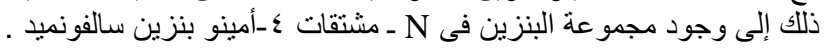

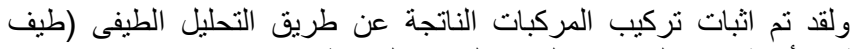
الكتلة ـ الأشعة تحت الحمر اء و الرنين النووى المغناطيسى) . 